\title{
Kernel Functions for Difference Operators of Ruijsenaars Type and Their Applications ${ }^{\star}$
}

\author{
Yasushi KOMORI ${ }^{\dagger}$, Masatoshi NOUMI ${ }^{\ddagger}$ and Jun’ichi SHIRAISHI ${ }^{\S}$ \\ $\dagger$ Graduate School of Mathematics, Nagoya University, Chikusa-Ku, Nagoya 464-8602, Japan \\ E-mail:komori@math.nagoya-u.ac.jp \\ $\ddagger$ Department of Mathematics, Kobe University, Rokko, Kobe 657-8501, Japan \\ E-mail:noumi@math.kobe-u.ac.jp \\ $\S$ Graduate School of Mathematical Sciences, University of Tokyo, \\ Komaba, Tokyo 153-8914, Japan \\ E-mail: shiraish@ms.u-tokyo.ac.jp
}

Received December 01, 2008, in final form April 30, 2009; Published online May 12, 2009 doi:10.3842/SIGMA.2009.054

\begin{abstract}
A unified approach is given to kernel functions which intertwine Ruijsenaars difference operators of type $A$ and of type $B C$. As an application of the trigonometric cases, new explicit formulas for Koornwinder polynomials attached to single columns and single rows are derived.
\end{abstract}

Key words: kernel function; Ruijsenaars operator; Koornwinder polynomial

2000 Mathematics Subject Classification: 81R12; 33D67

\section{Contents}

1 Introduction $\quad 2$

2 Kernel functions for Ruijsenaars operators $\quad 3$

2.1 Variations of the gamma function . . . . . . . . . . . . . . . . 3

2.2 Kernel functions of type $A \ldots \ldots \ldots \ldots \ldots \ldots$

2.3 Kernel functions of type $B C \ldots \ldots \ldots \ldots \ldots$

3 Derivation of kernel functions 10

3.1 Key identities . . . . . . . . . . . . . . . . . . . . . . . . . . . . . . . . . . . . . .

3.2 Case of type $A \ldots \ldots \ldots \ldots \ldots \ldots \ldots \ldots$

3.3 Case of type $B C \ldots \ldots \ldots \ldots \ldots \ldots \ldots$

3.4 Difference operators of Koornwinder type $\ldots \ldots \ldots \ldots$

4 Kernel functions for $q$-difference operators $\quad 19$

4.1 Kernel functions for Macdonald operators . . . . . . . . . . . . . . . . 19

4.2 Kernel functions for Koornwinder operators . . . . . . . . . . . . . . . . . . 20

* This paper is a contribution to the Proceedings of the Workshop "Elliptic Integrable Systems, Isomonodromy Problems, and Hypergeometric Functions" (July 21-25, 2008, MPIM, Bonn, Germany). The full collection is available at http://www.emis.de/journals/SIGMA/Elliptic-Integrable-Systems.html 
5 Application to Koornwinder polynomials $\quad 22$

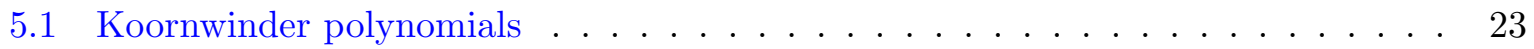

5.2 Case of a single column . . . . . . . . . . . . . . . . . . . 25

5.3 Case of a single row . . . . . . . . . . . . . . . . . . . 27

A Remarks on higher order difference operators $\quad 32$

B Comparison with [23] 33

B.1 Case of type $A \ldots \ldots \ldots \ldots \ldots \ldots \ldots$

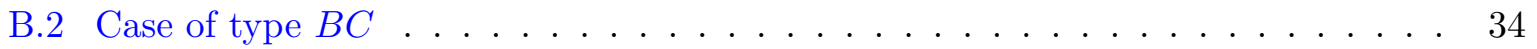

C $E_{r}(z ; a \mid t)$ and $H_{l}(z ; a \mid q, t)$ as interpolation polynomials $\quad 38$

$\begin{array}{ll}\text { References } & 40\end{array}$

\section{Introduction}

Let $\Phi(x ; y)$ be a meromorphic function on $\mathbb{C}^{m} \times \mathbb{C}^{n}$ in the variables $x=\left(x_{1}, \ldots, x_{m}\right)$ and $y=\left(y_{1}, \ldots, y_{n}\right)$, and consider two operators $\mathcal{A}_{x}, \mathcal{B}_{y}$ which act on meromorphic functions in $x$ and $y$, respectively. We say that $\Phi(x ; y)$ is a kernel function for the pair $\left(\mathcal{A}_{x}, \mathcal{B}_{y}\right)$ if it satisfies a functional equation of the form

$$
\mathcal{A}_{x} \Phi(x ; y)=\mathcal{B}_{y} \Phi(x ; y) \text {. }
$$

In the theory of Jack and Macdonald polynomials [14], certain explicit kernel functions play crucial roles in eigenfunction expansions and integral representations. Recently, kernel functions in this sense have been studied systematically by Langmann $[12,13]$ in the analysis of eigenfunctions for the elliptic quantum integrable systems of Calogero-Moser type, and by Ruijsenaars $[21,22,23]$ for the relativistic elliptic quantum integrable systems of Ruijsenaars-Schneider type.

In this paper we investigate two kinds of kernel functions, of Cauchy type and of dual Cauchy type, which intertwine pairs of Ruijsenaars difference operators. In the cases of elliptic difference operators, kernel functions of Cauchy type for the $\left(A_{n-1}, A_{n-1}\right)$ and $\left(B C_{n}, B C_{n}\right)$ cases were found by Ruijsenaars [22, 23]. Extending his result, we present kernel functions of Cauchy type, as well as those of dual Cauchy type, for the $\left(B C_{m}, B C_{n}\right)$ cases with arbitrary $m, n$ (under certain balancing conditions on the parameters in the elliptic cases). For the trigonometric difference operators of type $A$, kernel functions both of Cauchy and dual Cauchy types were already discussed by Macdonald [14]. Kernel functions of dual Cauchy type for the trigonometric $B C_{n}$ cases are due to Mimachi [15]. In this paper we develop a unified approach to kernel functions for Ruijsenaars operators of type $A$ and of type $B C$, with rational, trigonometric and elliptic coefficients, so as to cover all these known examples in the difference cases. We expect that our framework could be effectively applied to the study of eigenfunctions for difference operators of Ruijsenaars type.

As such an application of kernel functions in the trigonometric $B C$ cases, we derive new explicit formulas for Koornwinder polynomials attached to single columns and single rows. This provides with a direct construction of those special cases of the binomial expansion formula for the Koornwinder polynomials as studied by Okounkov [18] and Rains [19]. We also remark that, regarding explicit formulas for Macdonald polynomials attached to single rows of type $B, C, D$, some conjectures have been proposed by Lassalle [11]. The relationship between his conjectures and our kernel functions will be discussed in a separate paper.

Our main results on the kernel functions for Ruijsenaars difference operators of type $A$ and of type $B C$ will be formulated in Section 2. In Section 3 we give a unified proof for them on the 
basis of two key identities. After giving remarks in Section 4 on the passage to the $q$-difference operators of Macdonald and Koornwinder, as an application of our approach we present in Section 5 explicit formulas for Koornwinder polynomials attached to single columns and single rows. We also include three sections in Appendix. We will give some remarks in Appendix A on higher order difference operators, and in Appendix B make an explicit comparison of our kernel functions in the elliptic cases with those constructed by Ruijsenaars [23]. In Appendix C, we will give a proof of the fact that certain Laurent polynomials, which appear in our explicit formulas for Koornwinder polynomials attached to single columns and single rows, are special cases of the $B C_{m}$ interpolation polynomials of Okounkov [18].

\section{Kernel functions for Ruijsenaars operators}

\subsection{Variations of the gamma function}

In order to specify the class of Ruijsenaars operators which we shall discuss below, by the symbol $[u]$ we denote a nonzero entire function in one variable $u$, satisfying the following Riemann relation:

$$
\begin{gathered}
{[x+u][x-u][y+v][y-v]-[x+v][x-v][y+u][y-u]} \\
=[x+y][x-y][u+v][u-v]
\end{gathered}
$$

for any $x, y, u, v \in \mathbb{C}$. Under this condition, it is known that $[u]$ must be an odd function $([-u]=-[u]$, and hence $[0]=0)$, and the set $\Omega$ of all zeros of $[u]$ forms an additive subgroup of $\mathbb{C}$. Furthermore such functions are classified into the three categories, rational, trigonometric and elliptic, according to the rank of the additive subgroup $\Omega \subset \mathbb{C}$ of all zeros of $[u]$. In fact, up to multiplication by nonzero constants, $[u]$ coincides with one of the following three types of functions:

$$
\begin{array}{lll}
\text { (0) rational case: } & e\left(a u^{2}\right) u & (\Omega=0), \\
\text { (1) trigonometric case: } & e\left(a u^{2}\right) \sin \left(\pi u / \omega_{1}\right) & \left(\Omega=\mathbb{Z} \omega_{1}\right), \\
\text { (2) elliptic case: } & e\left(a u^{2}\right) \sigma(u ; \Omega) & \left(\Omega=\mathbb{Z} \omega_{1} \oplus \mathbb{Z} \omega_{2}\right),
\end{array}
$$

where $a \in \mathbb{C}$ and $e(u)=\exp (2 \pi \sqrt{-1} u)$. Also, $\sigma(x ; \Omega)$ denotes the Weierstrass sigma function

$$
\sigma(u ; \Omega)=u \prod_{\omega \in \Omega ; \omega \neq 0}\left(1-\frac{u}{\omega}\right) e^{\frac{u}{\omega}+\frac{u^{2}}{2 \omega^{2}}}
$$

associated with the period lattice $\Omega=\mathbb{Z} \omega_{1} \oplus \mathbb{Z} \omega_{2}$, generated by $\omega_{1}$, $\omega_{2}$ which are linearly independent over $\mathbb{R}$.

We start with some remarks on gamma functions associated with the function $[u]$. Fixing a nonzero scaling constant $\delta \in \mathbb{C}$, suppose that a nonzero meromorphic function $G(u \mid \delta)$ on $\mathbb{C}$ satisfies the difference equation

$$
G(u+\delta \mid \delta)=[u] G(u \mid \delta) \quad(u \in \mathbb{C}) .
$$

Such a function $G(u \mid \delta)$, determined up to multiplication by $\delta$-periodic functions, will be called a gamma function for $[u]$. We give typical examples of gamma functions in this sense for the rational, trigonometric and elliptic cases.

(0) Rational case: For any $\delta \in \mathbb{C}(\delta \neq 0)$, the meromorphic function

$$
G(u \mid \delta)=\delta^{u / \delta} \Gamma(u / \delta),
$$

defined with the Euler gamma function $\Gamma(u)$, is a gamma function for $[u]=u$. 
(1) Trigonometric case: We set $z=e\left(u / \omega_{1}\right)$ and $q=e\left(\delta / \omega_{1}\right)$, and suppose that $\operatorname{Im}\left(\delta / \omega_{1}\right)>0$ so that $|q|<1$. We now consider the function

$$
[u]=z^{\frac{1}{2}}-z^{-\frac{1}{2}}=2 \sqrt{-1} \sin \left(\pi u / \omega_{1}\right) .
$$

For this $[u]$ two meromorphic functions

$$
G_{-}(u \mid \delta)=\frac{e\left(-\frac{\delta}{2 \omega_{1}}\left(\begin{array}{c}
u / \delta \\
2
\end{array}\right)\right)}{(z ; q)_{\infty}}, \quad G_{+}(u \mid \delta)=e\left(\frac{\delta}{2 \omega_{1}}\left(\begin{array}{c}
u / \delta \\
2
\end{array}\right)\right)(q / z ; q)_{\infty}
$$

where $(z ; q)_{\infty}=\prod_{i=0}^{\infty}\left(1-q^{i} z\right)$, satisfy the difference equations

$$
G_{-}(u+\delta \mid \delta)=-[u] G_{-}(u \mid \delta), \quad G_{+}(u+\delta \mid \delta)=[u] G_{+}(u \mid \delta),
$$

respectively. Namely, for $\epsilon= \pm, G_{\epsilon}(u \mid \delta)$ is a gamma function for $\epsilon[u]$. (Note that the quadratic function $\left(\begin{array}{l}u \\ 2\end{array}\right)=\frac{1}{2} u(u-1)$ satisfies $\left(\begin{array}{c}u+1 \\ 2\end{array}\right)=\left(\begin{array}{l}u \\ 2\end{array}\right)+u$.) Another set of gamma functions for $\epsilon[u]$ of this case is given by

$$
G_{+}(u \mid \delta)=\frac{\left(q^{\frac{1}{2}} z^{-\frac{1}{2}} ; q^{\frac{1}{2}}\right)_{\infty}}{\left(-z^{\frac{1}{2}} ; q^{\frac{1}{2}}\right)_{\infty}}, \quad G_{-}(u \mid \delta)=\frac{\left(-q^{\frac{1}{2}} z^{-\frac{1}{2}} ; q^{\frac{1}{2}}\right)_{\infty}}{\left(z^{\frac{1}{2}} ; q^{\frac{1}{2}}\right)_{\infty}}
$$

by means of the infinite products with base $q^{\frac{1}{2}}$.

(2) Elliptic case: Let $p, q$ be nonzero complex numbers with $|p|<1,|q|<1$. Then the Ruijsenaars elliptic gamma function

$$
\Gamma(z ; p, q)=\frac{(p q / z ; p, q)_{\infty}}{(z ; p, q)_{\infty}}, \quad(z ; p, q)_{\infty}=\prod_{i, j=0}^{\infty}\left(1-p^{i} q^{j} z\right)
$$

satisfies the $q$-difference equation

$$
\Gamma(q z ; p, q)=\theta(z ; p) \Gamma(z ; p, q), \quad \theta(z ; p)=(z ; p)_{\infty}(p / z ; p)_{\infty}
$$

Note also $\Gamma(p q / z ; p, q)=\Gamma(z ; p, q)^{-1}$. We set $p=e\left(\omega_{2} / \omega_{1}\right), q=e\left(\delta / \omega_{1}\right)$ and $z=e\left(u / \omega_{1}\right)$, and suppose that $\operatorname{Im}\left(\omega_{2} / \omega_{1}\right)>0, \operatorname{Im}\left(\delta / \omega_{1}\right)>0$ so that $|p|<1,|q|<1$. Instead of $\theta(z ; p)$ above, we consider the function

$$
[u]=-z^{-\frac{1}{2}} \theta(z ; p)=2 \sqrt{-1} \sin \left(\pi u / \omega_{1}\right)(p z ; p)_{\infty}(p / z ; p)_{\infty}
$$

which is a constant multiple of the odd Jacobi theta function with modulus $\omega_{2} / \omega_{1}$, so that $[u]$ should satisfy the Riemann relation. Then the two meromorphic functions

$$
\begin{aligned}
& G_{-}(u \mid \delta)=e\left(-\frac{\delta}{2 \omega_{1}}\left(\begin{array}{c}
u / \delta \\
2
\end{array}\right)\right) \Gamma(z ; p, q)=e\left(-\frac{\delta}{2 \omega_{1}}\left(\begin{array}{c}
u / \delta \\
2
\end{array}\right)\right) \frac{(p q / z ; p, q)_{\infty}}{(z ; p, q)_{\infty}}, \\
& G_{+}(u \mid \delta)=e\left(\frac{\delta}{2 \omega_{1}}\left(\begin{array}{c}
u / \delta \\
2
\end{array}\right)\right) \Gamma(p z ; p, q)=e\left(\frac{\delta}{2 \omega_{1}}\left(\begin{array}{c}
u / \delta \\
2
\end{array}\right)\right) \frac{(q / z ; p, q)_{\infty}}{(p z ; p, q)_{\infty}} .
\end{aligned}
$$

satisfy the difference equations

$$
G_{-}(u+\delta \mid \delta)=-[u] G_{-}(u \mid \delta), \quad G_{+}(u+\delta \mid \delta)=[u] G_{+}(u \mid \delta),
$$

respectively. Another set of gamma functions for $\epsilon[u]$ is given by

$$
G_{\epsilon}(u \mid \delta)=\theta\left(\epsilon z^{\frac{1}{2}} ; q^{\frac{1}{2}}\right) \Gamma(z ; p, q)=\Gamma\left(\epsilon p^{\frac{1}{2}} z^{\frac{1}{2}} ; p^{\frac{1}{2}}, q^{\frac{1}{2}}\right) \Gamma\left(-\epsilon z^{\frac{1}{2}} ; p^{\frac{1}{2}}, q^{\frac{1}{2}}\right) \quad(\epsilon= \pm) .
$$

In the limit as $p \rightarrow 0$, these examples recover the previous ones in the trigonometric case.

We remark that, if $G(u \mid \delta)$ is a gamma function for $[u]$, then $G(\delta-u \mid \delta)^{-1}$ is a gamma function for $-[u]$. Also, when we transform $[u]$ to $[u]^{\prime}=c e\left(a u^{2}\right)[u]$, a gamma function for $[u]^{\prime}$ is obtained for instance as $G^{\prime}(u \mid \delta)=c^{u / \delta} e(a p(u)) G(u \mid \delta)$, where $p(u)=\frac{1}{3 \delta} u^{3}-\frac{1}{2} u^{2}+\frac{\delta}{6} u$. 


\subsection{Kernel functions of type $A$}

The elliptic difference operator which we will discuss below was introduced by Ruijsenaars [20] together with the commuting family of higher order difference operators. In order to deal with rational, trigonometric and elliptic cases in a unified manner, we formulate our results for this class of (first order) difference operators in terms of an arbitrary function $[u]$ satisfying the Riemann relation. (As to the commuting family of higher order difference operators, we will give some remarks later in Appendix A.)

Fix a nonzero entire function $[u]$ satisfying the Riemann relation (2.1). For type $A$, we consider the difference operator

$$
D_{x}^{(\delta, \kappa)}=\sum_{i=1}^{m} A_{i}(x ; \kappa) T_{x_{i}}^{\delta}, \quad A_{i}(x ; \kappa)=\prod_{1 \leq j \leq m ; j \neq i} \frac{\left[x_{i}-x_{j}+\kappa\right]}{\left[x_{i}-x_{j}\right]}
$$

in $m$ variables $x=\left(x_{1}, \ldots, x_{m}\right)$, where $\delta, \kappa \in \mathbb{C}$ are complex parameters $\notin \Omega$, and $T_{x_{i}}^{\delta}$ stands for the $\delta$-shift operator

$$
T_{x_{i}}^{\delta} f\left(x_{1}, \ldots, x_{m}\right)=f\left(x_{1}, \ldots, x_{i}+\delta, \ldots, x_{m}\right) \quad(i=1, \ldots, m) .
$$

Note that this operator remains invariant if one replaces the function $[u]$ with its multiple by any nonzero constant.

By taking a gamma function $G(u \mid \delta)$ for (any constant multiple of) $[u]$ as in (2.2), we define a function $\Phi_{A}(x ; y \mid \delta, \kappa)$ by

$$
\Phi_{A}(x ; y \mid \delta, \kappa)=\prod_{j=1}^{m} \prod_{l=1}^{n} \frac{G\left(x_{j}+y_{l}+v-\kappa \mid \delta\right)}{G\left(x_{j}+y_{l}+v \mid \delta\right)}
$$

with an extra parameter $v$. We also consider the function

$$
\Psi_{A}(x ; y)=\prod_{j=1}^{m} \prod_{l=1}^{n}\left[x_{j}-y_{l}+v\right] .
$$

These two functions $\Phi_{A}(x ; y \mid \delta, \kappa)$ and $\Psi_{A}(x ; y)$ are kernel functions of Cauchy type and of dual Cauchy type for this case, respectively.

Theorem 2.1. Let $[u]$ be any nonzero entire function satisfying the Riemann relation.

(1) If $m=n$, then the function $\Phi_{A}(x ; y \mid \delta, \kappa)$ defined as (2.5) satisfies the functional equation

$$
D_{x}^{(\delta, \kappa)} \Phi_{A}(x ; y \mid \delta, \kappa)=D_{y}^{(\delta, \kappa)} \Phi_{A}(x ; y \mid \delta, \kappa) .
$$

(2) Under the balancing condition $m \kappa+n \delta=0$, the function $\Psi_{A}(x ; y)$ defined as (2.6) satisfies the functional equation

$$
[\kappa] D_{x}^{(\delta, \kappa)} \Psi_{A}(x ; y)+[\delta] D_{y}^{(\kappa, \delta)} \Psi_{A}(x ; y)=0 .
$$

Statement (1) of Theorem 2.1 is due to Ruijsenaars [22, 23]. (See Appendix B.1 for an explicit comparison between $\Phi_{A}(x ; y)$ and Ruijsenaars' kernel function of [23].) In the scope of the present paper, the balancing conditions ( $m=n$ in (1), and $m \kappa+n \delta=0$ in (2)) seem to be essential in the elliptic cases. In the context of elliptic differential operators of Calogero-Moser type, however, Langmann [13] has found a natural generalization of the kernel identities of Cauchy type, which include the differentiation with respect to the elliptic modulus, to arbitrary pair $(m, n)$. It would be a intriguing problem to find a generalization of this direction for elliptic difference operators of Ruijsenaars type.

In trigonometric and rational cases, these functions $\Phi_{A}(x ; y \mid \delta, \kappa)$ and $\Psi_{A}(x ; y)$ satisfy more general functional equations without balancing conditions. 
Theorem 2.2. Suppose that $[u]$ is a constant multiple of $\sin \left(\pi u / \omega_{1}\right)$ or $u$.

(1) For arbitrary $m$ and $n$, the function $\Phi_{A}(x ; y \mid \delta, \kappa)$ satisfies the functional equation

$$
D_{x}^{(\delta, \kappa)} \Phi_{A}(x ; y \mid \delta, \kappa)-D_{y}^{(\delta, \kappa)} \Phi_{A}(x ; y \mid \delta, \kappa)=\frac{[(m-n) \kappa]}{[\kappa]} \Phi_{A}(x ; y \mid \delta, \kappa) .
$$

(2) The function $\Psi_{A}(x ; y)$ satisfies the functional equation

$$
[\kappa] D_{x}^{(\delta, \kappa)} \Psi_{A}(x ; y)+[\delta] D_{y}^{(\kappa, \delta)} \Psi_{A}(x ; y)=[m \kappa+n \delta] \Psi_{A}(x ; y) .
$$

These results for the trigonometric (and rational) cases are essentially contained in the discussion of Macdonald [14].

A unified proof of Theorems 2.1 and 2.2 will be given in Section 3. We will also explain in Section 4 how Theorem 2.2 is related with the theory of Macdonald polynomials.

\subsection{Kernel functions of type $B C$}

The (first-order) elliptic difference operator of type $B C$ was first proposed by van Diejen [2]. It is also known by Komori-Hikami [9] that it admits a commuting family of higher order difference operators. In the following we use the expression of the first-order difference operator due to [9], with modification in terms of $[u]$. (In Appendix B, we will give some remarks on the comparison of our difference operator with other expressions in the literature.)

For type $B C$, we consider difference operators of the form

$$
E_{x}^{(\mu \mid \delta, \kappa)}=\sum_{i=1}^{m} A_{i}^{+}(x ; \mu \mid \delta, \kappa) T_{x_{i}}^{\delta}+\sum_{i=1}^{m} A_{i}^{-}(x ; \mu \mid \delta, \kappa) T_{x_{i}}^{-\delta}+A^{0}(x ; \mu \mid \delta, \kappa)
$$

including $2 \rho$ parameters $\mu=\left(\mu_{1}, \ldots, \mu_{2} \rho\right)$ besides $(\delta, \kappa)$, where $\rho=1,2$ or 4 according as $\operatorname{rank} \Omega=0,1$, or 2 . In the trigonometric and rational cases of type $B C$, we assume that the function $[u]$ does not contain exponential factors. Namely, we assume that $[u]$ is a constant multiple of one of the functions

$$
u(\rho=1), \quad \sin \left(\pi u / \omega_{1}\right) \quad(\rho=2), \quad e\left(a u^{2}\right) \sigma(u ; \Omega) \quad(\rho=4) .
$$

In each case we define $\omega_{1}, \ldots, \omega_{\rho} \in \Omega$ as

$$
\begin{array}{lll}
\text { (0) rational case: } & \Omega=0, & \omega_{1}=0, \\
\text { (1) trigonometric case: } & \Omega=\mathbb{Z} \omega_{1}, & \omega_{2}=0, \\
\text { (2) elliptic case: } & \Omega=\mathbb{Z} \omega_{1} \oplus \mathbb{Z} \omega_{2}, & \omega_{3}=-\omega_{1}-\omega_{2}, \quad \omega_{4}=0 .
\end{array}
$$

Then the quasi-periodicity of $[u]$ is described as

$$
\left[u+\omega_{r}\right]=\epsilon_{r} e\left(\eta_{r}\left(u+\frac{1}{2} \omega_{r}\right)\right)[u] \quad(r=1, \ldots, \rho)
$$

for some $\eta_{r} \in \mathbb{C}$ and $\epsilon_{r}= \pm 1$. In the trigonometric and rational cases (without exponential factors), one can simply take $\eta_{r}=0(r=1, \ldots, \rho)$. Note also that $[u]$ admits the duplication formula of the form

$$
[2 u]=2[u] \prod_{s=1}^{\rho-1} \frac{\left[u-\frac{1}{2} \omega_{s}\right]}{\left[-\frac{1}{2} \omega_{s}\right]}, \quad \frac{[2 u+c]}{[2 u]}=\prod_{s=1}^{\rho} \frac{\left[u+\frac{1}{2}\left(c-\omega_{s}\right)\right]}{\left[u-\frac{1}{2} \omega_{s}\right]} .
$$


(In the trigonometric and rational cases, these formulas fail for $[u]$ containing nontrivial exponential factors $e\left(a u^{2}\right)$.) In relation to the parameters $\mu=\left(\mu_{1}, \ldots, \mu_{2 \rho}\right)$, we introduce

$$
c^{(\mu \mid \delta, \kappa)}=\sum_{s=1}^{2 \rho} \mu_{s}-\frac{\rho}{2}(\delta+\kappa)+\sum_{s=1}^{\rho} \omega_{s} .
$$

This parameter is related to quasi-periodicity of the coefficients of the Ruijsenaars operator, and plays a crucial role in various places of our argument. Note that the last term $\sum_{s=1}^{\rho} \omega_{s}$ is nontrivial only in the trigonometric case: it is $\omega_{1}$ when $\rho=2$, and 0 when $\rho=1,4$.

With these data, we define the coefficients of the Ruijsenaars operator $E_{x}^{(\mu \mid \delta, \kappa)}$ of type $B C$ as follows:

$$
\begin{aligned}
A_{i}^{+}(x ; \mu \mid \delta, \kappa) & =\frac{\prod_{s=1}^{2 \rho}\left[x_{i}+\mu_{s}\right]}{\prod_{s=1}^{\rho}\left[x_{i}-\frac{1}{2} \omega_{s}\right]\left[x_{i}+\frac{1}{2}\left(\delta-\omega_{s}\right)\right]} \prod_{1 \leq j \leq m ; j \neq i} \frac{\left[x_{i} \pm x_{j}+\kappa\right]}{\left[x_{i} \pm x_{j}\right]}, \\
A_{i}^{-}(x ; \mu \mid \delta, \kappa) & =A_{i}^{+}(-x ; \mu \mid \delta, \kappa) \quad(i=1, \ldots, m),
\end{aligned}
$$

with an abbreviated notation $[x \pm y]=[x+y][x-y]$ of products, and

$$
\begin{aligned}
A^{0}(x ; \mu \mid \delta, \kappa) & =\sum_{r=1}^{\rho} A_{r}^{0}(x ; \mu \mid \delta, \kappa), \\
A_{r}^{0}(x ; \mu \mid \delta, \kappa) & =\frac{e\left(-\left(m \kappa+\frac{1}{2} c^{(\mu \mid \delta, \kappa)}\right) \eta_{r}\right) \prod_{s=1}^{2 \rho}\left[\frac{1}{2}\left(\omega_{r}-\delta\right)+\mu_{s}\right]}{[\kappa] \prod_{s \neq r}\left[\frac{1}{2}\left(\omega_{r}-\omega_{s}\right)\right] \prod_{s=1}^{\rho}\left[\frac{1}{2}\left(\omega_{r}-\omega_{s}+\kappa-\delta\right)\right]} \frac{\left[\frac{1}{2}\left(\omega_{r}-\delta\right) \pm x_{j}+\kappa\right]}{\left[\frac{1}{2}\left(\omega_{r}-\delta\right) \pm x_{j}\right]} .
\end{aligned}
$$

This operator $E_{x}^{(\mu \mid \delta, \kappa)}$ is one of the expressions for the first-order Ruijsenaars operator of type $B C_{m}$ due to Komori-Hikami [9, (4.21)]. We remark that this difference operator $E_{x}^{(\mu \mid \delta, \kappa)}$ has symmetry

$$
E_{x}^{(\mu \mid-\delta,-\kappa)}=E_{x}^{(-\mu \mid \delta, \kappa)}
$$

with respect to the sign change of parameters. (This property can be verified directly by using the quasi-periodicity of $[u]$ and the fact that $\prod_{s=1}^{\rho} \epsilon_{s}=1,-1,-1$ according as $\rho=1,2,4$.) Note also that it remains invariant if one replace the function $[u]$ with its multiple by a nonzero constant. By the duplication formula (2.9), one can also rewrite the operator $E_{x}^{(\mu \mid \delta, \kappa)}$ in the form

$$
\begin{aligned}
\frac{1}{4} \prod_{s=1}^{\rho-1}\left[\frac{1}{2} \omega_{s}\right]^{2} E_{x}^{(\mu \mid \delta, \kappa)}= & \sum_{i=1}^{m} \frac{\prod_{s=1}^{2 \rho}\left[x_{i}+\mu_{s}\right]}{\left[2 x_{i}\right]\left[2 x_{i}+\delta\right]} \prod_{1 \leq j \leq m ; j \neq i} \frac{\left[x_{i} \pm x_{j}+\kappa\right]}{\left[x_{i} \pm x_{j}\right]} T_{x_{i}}^{\delta} \\
& +\sum_{i=1}^{m} \frac{\prod_{s=1}^{2 \rho}\left[-x_{i}+\mu_{s}\right]}{\left[-2 x_{i}\right]\left[-2 x_{i}+\delta\right]} \prod_{1 \leq j \leq m ; j \neq i} \frac{\left[-x_{i} \pm x_{j}+\kappa\right]}{\left[-x_{i} \pm x_{j}\right]} T_{x_{i}}^{-\delta} \\
& +\sum_{r=1}^{\rho} K_{r} \frac{\prod_{s=1}^{2 \rho}\left[\frac{1}{2}\left(\omega_{r}-\delta\right)+\mu_{s}\right]}{2[\kappa][\kappa-\delta]} \prod_{j=1}^{m} \frac{\left[\frac{1}{2}\left(\omega_{r}-\delta\right) \pm x_{j}+\kappa\right]}{\left[\frac{1}{2}\left(\omega_{r}-\delta\right) \pm x_{j}\right]},
\end{aligned}
$$

where $K_{r}=e\left(-\left(\omega_{r}+(m+1) \kappa-\delta+\frac{1}{2} c^{(\mu \mid \delta, \kappa)}\right) \eta_{r}\right)$. 
By using any gamma function $G(u \mid \delta)$ for (a constant multiple of) [u], we define a function $\Phi_{B C}(x ; y \mid \delta, \kappa)$ of Cauchy type, either by

$$
\Phi_{B C}(x ; y \mid \delta, \kappa)=\prod_{j=1}^{m} \prod_{l=1}^{n} \frac{G\left(x_{j} \pm y_{l}+\frac{1}{2}(\delta-\kappa) \mid \delta\right)}{G\left(x_{j} \pm y_{l}+\frac{1}{2}(\delta+\kappa) \mid \delta\right)},
$$

or by

$$
\Phi_{B C}(x ; y \mid \delta, \kappa)=\prod_{j=1}^{m} \prod_{l=1}^{n} \prod_{\epsilon_{1}, \epsilon_{2}= \pm} G\left(\epsilon_{1} x_{j}+\epsilon_{2} y_{l}+\frac{1}{2}(\delta-\kappa) \mid \delta\right) .
$$

Note that the function (2.15) differs from (2.14), only by a multiplicative factor which is $\delta$ periodic in all the variables $x_{j}(j=1, \ldots, m)$ and $y_{l}(l=1, \ldots, n)$. We also introduce the function

$$
\Psi_{B C}(x ; y)=\prod_{j=1 l=1}^{m} \prod_{l}^{n}\left[x_{j} \pm y_{l}\right]
$$

of dual Cauchy type.

Theorem 2.3. Suppose that $[u]$ is a constant multiple of any function in (2.8).

(1) Under the balancing condition

$$
2(m-n) \kappa+c^{(\mu \mid \delta, \kappa)}=0,
$$

the function $\Phi_{B C}(x ; y \mid \delta, \kappa)$ defined as (2.14) or (2.15) satisfies the functional equation

$$
E_{x}^{(\mu \mid \delta, \kappa)} \Phi_{B C}(x ; y \mid \delta, \kappa)=E_{y}^{(\nu \mid \delta, \kappa)} \Phi_{B C}(x ; y \mid \delta, \kappa),
$$

where the parameters $\nu=\left(\nu_{1}, \ldots, \nu_{2} \rho\right)$ for the $y$ variables are defined by

$$
\nu_{r}=\frac{1}{2}(\delta+\kappa)-\mu_{r} \quad(r=1, \ldots, 2 \rho) .
$$

(2) Under the balancing condition

$$
2 m \kappa+2 n \delta+c^{(\mu \mid \delta, \kappa)}=0
$$

the function $\Psi_{B C}(x ; y)$ defined as (2.16) satisfies the functional equation

$$
[\kappa] E_{x}^{(\mu \mid \delta, \kappa)} \Psi_{B C}(x ; y)+[\delta] E_{y}^{(\mu \mid \kappa, \delta)} \Psi_{B C}(x ; y)=0 .
$$

Statement (1) for the cases $m=n$ is due to Ruijsenaars [21, 23]. An explicit comparison will be made in Appendix B.2 between our $\Phi_{B C}(x ; y \mid \delta, \kappa)$ and Ruijsenaars' kernel function of type $B C$ in [23].

In the case of 0 variables, the Ruijsenaars operator $E_{x}^{(\mu \mid \delta, \kappa)}$ with $m=0$ reduces to the multiplication operator by the constant

$$
C^{(\mu \mid \delta, \kappa)}=\sum_{r=1}^{\rho} \frac{e\left(-\frac{1}{2} c^{(\mu \mid \delta, \kappa)} \eta_{r}\right) \prod_{s=1}^{2 \rho}\left[\frac{1}{2}\left(\omega_{r}-\delta\right)+\mu_{s}\right]}{\prod_{s \neq r}\left[\frac{1}{2}\left(\omega_{r}-\omega_{s}\right)\right] \prod_{s=1}^{\rho}\left[\frac{1}{2}\left(\omega_{r}-\omega_{s}+\kappa-\delta\right)\right]} .
$$


We remark that Theorem 2.3, (2) for $n=0$ implies that, when $2 m \kappa+c^{(\mu \mid \delta, \kappa)}=0$, the constant function 1 is an eigenfunction for $E_{x}^{(\mu \mid \delta, \kappa)}$ :

$$
[\kappa] E_{x}^{(\mu \mid \delta, \kappa)}(1)=-[\delta] C^{(\mu \mid \kappa, \delta)} .
$$

(Statement (1) for $n=0$ gives the same formula since $C^{(\nu \mid \delta, \kappa)}=-[\delta] C^{(\mu \mid \kappa, \delta)} /[\kappa]$.)

In the trigonometric and rational cases, these functions $\Phi_{B C}(x ; y \mid \delta, \kappa)$ and $\Psi_{B C}(x ; y)$ satisfy the following functional equations without balancing conditions.

Theorem 2.4. Suppose that $[u]$ is a constant multiple of $\sin \left(\pi u / \omega_{1}\right)(\rho=2)$ or $u(\rho=1)$.

(1) The function $\Phi_{B C}(x ; y \mid \delta, \kappa)$ satisfies the functional equation

$$
\begin{gathered}
{[\kappa] E_{x}^{(\mu \mid \delta, \kappa)} \Phi_{B C}(x ; y \mid \delta, \kappa)-[\kappa] E_{y}^{(\nu \mid \delta, \kappa)} \Phi_{B C}(x ; y \mid \delta, \kappa)} \\
=\left[2(m-n) \kappa+c^{(\mu \mid \delta, \kappa)}\right] \Phi_{B C}(x ; y \mid \delta, \kappa),
\end{gathered}
$$

where $\nu_{r}=\frac{1}{2}(\delta+\kappa)-\mu_{r}(r=1, \ldots, 2 \rho)$.

(2) The function $\Psi_{B C}(x ; y)$ satisfies the functional equation

$$
[\kappa] E_{x}^{(\mu \mid \delta, \kappa)} \Psi_{B C}(x ; y)+[\delta] E_{y}^{(\mu \mid \kappa, \delta)} \Psi_{B C}(x ; y)=\left[2 m \kappa+2 n \delta+c^{(\mu \mid \delta, \kappa)}\right] \Psi_{B C}(x ; y) .
$$

As a special case $n=0$ of this theorem, we see that the constant function 1 is a eigenfunction of $E_{x}^{(\mu \mid \delta, \kappa)}$ for arbitrary values of the parameters. Theorems 2.3 and 2.4 will be proved in Section 3.

In the trigonometric and rational $B C$ cases, it is convenient to introduce another difference operator

$$
D_{x}^{(\mu \mid \delta, \kappa)}=\sum_{i=1}^{m} A_{i}^{+}(x ; \mu \mid \delta, \kappa)\left(T_{x_{i}}^{\delta}-1\right)+\sum_{i=1}^{m} A_{i}^{-}(x ; \mu \mid \delta, \kappa)\left(T_{x_{i}}^{-\delta}-1\right)
$$

with the same coefficients $A_{i}^{\epsilon}(x ; \mu \mid \delta, \kappa)(i=1, \ldots, m ; \epsilon= \pm)$ as those of the Ruijsenaars operator $E_{x}^{(\mu \mid \delta, \kappa)}$. In the trigonometric case, this operator $D_{x}^{(\mu \mid \delta, \kappa)}$ is a constant multiple of Koornwinder's $q$-difference operator expressed in terms of additive variables. By using the relation

$$
D_{x}^{(\mu \mid \delta, \kappa)}=E_{x}^{(\mu \mid \delta, \kappa)}-E_{x}^{(\mu \mid \delta, \kappa)}(1),
$$

one can rewrite the functional equations in Theorem 2.4 into those for $D_{x}^{(\mu \mid \delta, \kappa)}$.

Theorem 2.5. Suppose that $[u]=2 \sqrt{-1} \sin \left(\pi u / \omega_{1}\right), \rho=2($ resp. $[u]=u, \rho=1)$.

(1) The function $\Phi_{B C}(x ; y \mid \delta, \kappa)$ satisfies the functional equation

$$
\begin{aligned}
& {[\kappa] D_{x}^{(\mu \mid \delta, \kappa)} \Phi_{B C}(x ; y \mid \delta, \kappa)-[\kappa] D_{y}^{(\nu \mid \delta, \kappa)} \Phi_{B C}(x ; y \mid \delta, \kappa)} \\
& \quad=[m \kappa][-n \kappa]\left[(m-n) \kappa+c^{(\mu \mid \delta, \kappa)}\right] \Phi_{B C}(x ; y \mid \delta, \kappa) \quad(\text { resp. }=0),
\end{aligned}
$$

where $\nu_{r}=\frac{1}{2}(\delta+\kappa)-\mu_{r}(r=1, \ldots, 2 \rho)$.

(2) For arbitrary $m, n$, the function $\Psi_{B C}(x ; y)$ satisfies the functional equation

$$
\begin{aligned}
& {[\kappa] D_{x}^{(\mu \mid \delta, \kappa)} \Psi_{B C}(x ; y)+[\delta] D_{y}^{(\mu \mid \kappa, \delta)} \Psi_{B C}(x ; y)} \\
& \quad=[m \kappa][n \delta]\left[m \kappa+n \delta+c^{(\mu \mid \delta, \kappa)}\right] \Psi_{B C}(x ; y) \quad(\text { resp. }=0) .
\end{aligned}
$$

Statement (2) of Theorem 2.5 recovers a main result of Mimachi [15]. A proof of Theorem 2.5 will be given in Section 3.4. Also, we will explain in Section 4 how Theorem 2.5 is related to the theory of Koornwinder polynomials. 


\section{Derivation of kernel functions}

\subsection{Key identities}

We start with a functional identity which decomposes a product of functions expressed by $[u]$ into partial fractions.

Proposition 3.1. Let $[u]$ be any nonzero entire function satisfying the Riemann relation. Then, for variables $z,\left(x_{1}, \ldots, x_{N}\right)$, and parameters $\left(c_{1}, \ldots, c_{N}\right)$, we have

$$
[c] \prod_{j=1}^{N} \frac{\left[z-x_{j}+c_{j}\right]}{\left[z-x_{j}\right]}=\sum_{i=1}^{N}\left[c_{i}\right] \frac{\left[z-x_{i}+c\right]}{\left[z-x_{i}\right]} \prod_{1 \leq j \leq N ; j \neq i} \frac{\left[x_{i}-x_{j}+c_{j}\right]}{\left[x_{i}-x_{j}\right]},
$$

where $c=c_{1}+\cdots+c_{N}$.

This identity can be proved by the induction on the number of factors by using the Riemann relation for $[u]$. Note that, by setting $y_{j}=x_{j}-c_{j}$, this identity can also be rewritten as

$$
[c] \prod_{j=1}^{N} \frac{\left[z-y_{j}\right]}{\left[z-x_{j}\right]}=\sum_{i=1}^{N}\left[x_{i}-y_{i}\right] \frac{\left[z-x_{i}+c\right]}{\left[z-x_{i}\right]} \prod_{1 \leq j \leq N ; j \neq i} \frac{\left[x_{i}-y_{j}\right]}{\left[x_{i}-x_{j}\right]}
$$

where $c=\sum_{j=1}^{N}\left(x_{j}-y_{j}\right)$.

From this proposition, we obtain the following lemma, which provides key identities for our proof of kernel relations.

Lemma 3.1. Consider $N$ variables $x_{1}, \ldots, x_{N}$ and $N$ complex parameters $c_{1}, \ldots, c_{N}$.

(1) Let $[u]$ be any nonzero function satisfying the Riemann relation. Then under the balancing condition $\sum_{j=1}^{N} c_{j}=0$, we have an identity

$$
\sum_{i=1}^{N}\left[c_{i}\right] \prod_{1 \leq j \leq N ; j \neq i} \frac{\left[x_{i}-x_{j}+c_{j}\right]}{\left[x_{i}-x_{j}\right]}=0
$$

as a meromorphic function in $\left(x_{1}, \ldots, x_{N}\right)$.

(2) Suppose that $[u]$ is a constant multiple of $\sin \left(\pi u / \omega_{1}\right)$ or $u$. Then for any $c_{1}, \ldots, c_{N} \in \mathbb{C}$, we have an identity

$$
\sum_{i=1}^{N}\left[c_{i}\right] \prod_{1 \leq j \leq N ; j \neq i} \frac{\left[x_{i}-x_{j}+c_{j}\right]}{\left[x_{i}-x_{j}\right]}=\left[\sum_{i=1}^{N} c_{i}\right]
$$

as a meromorphic function in $\left(x_{1}, \ldots, x_{N}\right)$.

When $[z]=\sin \left(\pi z / \omega_{1}\right)$, we have $[z+a] /[z+b] \rightarrow e\left(-(a-b) / 2 \omega_{1}\right)$ as $\operatorname{Im}\left(z / \omega_{1}\right) \rightarrow \infty$. This implies in (3.1)

$$
\prod_{j=1}^{N} \frac{\left[z-x_{j}+c_{j}\right]}{\left[z-x_{j}\right]} \rightarrow e\left(-c / 2 \omega_{1}\right), \quad \frac{\left[z-x_{i}+c\right]}{\left[z-x_{i}\right]} \rightarrow e\left(-c / 2 \omega_{1}\right) \quad(i=1, \ldots, m),
$$

as $\operatorname{Im}\left(z / \omega_{1}\right) \rightarrow \infty$. Hence we obtain (3.3) from (3.1). In the rational case $[z]=z$, formula (3.3) is derived by a simple limiting procedure $z \rightarrow \infty$. 


\subsection{Case of type $A$}

We apply the key identities (3.2) and (3.3) for studying kernel functions. For two sets of variables $x=\left(x_{1}, \ldots, x_{m}\right)$ and $y=\left(y_{1}, \ldots, y_{n}\right)$, we consider the following meromorphic function:

$$
F(z)=\prod_{j=1}^{m} \frac{\left[z-x_{j}+\kappa\right]}{\left[z-x_{j}\right]} \prod_{l=1}^{n} \frac{\left[z-y_{l}+v+\lambda\right]}{\left[z-y_{l}+v\right]}
$$

where $\kappa, \lambda$ and $v$ are complex parameters. Then by Proposition 3.1 this function $F(z)$ is expanded as

$$
\begin{aligned}
{[c] F(z)=} & {[\kappa] \sum_{i=1}^{m} \frac{\left[z-x_{i}+c\right]}{\left[z-x_{i}\right]} \prod_{1 \leq j \leq m ; j \neq i} \frac{\left[x_{i}-x_{j}+\kappa\right]}{\left[x_{i}-x_{j}\right]} \prod_{l=1}^{n} \frac{\left[x_{i}-y_{l}+v+\lambda\right]}{\left[x_{i}-y_{l}+v\right]} } \\
& +[\lambda] \sum_{k=1}^{n} \frac{\left[z-y_{k}+v+c\right]}{\left[z-y_{k}+v\right]} \prod_{1 \leq l \leq n ; l \neq k} \frac{\left[y_{k}-y_{l}+\lambda\right]}{\left[y_{k}-y_{l}\right]} \prod_{j=1}^{m} \frac{\left[y_{k}-x_{j}-v+\kappa\right]}{\left[y_{k}-x_{j}-v\right]} \\
= & {[\kappa] \sum_{i=1}^{m} \frac{\left[z-x_{i}+c\right]}{\left[z-x_{i}\right]} A_{i}(x ; \kappa) \prod_{l=1}^{n} \frac{\left[x_{i}-y_{l}+v+\lambda\right]}{\left[x_{i}-y_{l}+v\right]} } \\
& +[\lambda] \sum_{k=1}^{n} \frac{\left[z-y_{k}+v+c\right]}{\left[z-y_{k}+v\right]} A_{k}(y ; \lambda) \prod_{j=1}^{m} \frac{\left[y_{k}-x_{j}-v+\kappa\right]}{\left[y_{k}-x_{j}-v\right]},
\end{aligned}
$$

where $c=m \kappa+n \lambda$, with the coefficients $A_{i}(x ; \kappa), A_{k}(y ; \lambda)$ of Ruijsenaars operators in $x$ variables and $y$ variables. By Lemma 3.1, under the balancing condition $c=m \kappa+n \lambda=0$, we have

$$
[\kappa] \sum_{i=1}^{m} A_{i}(x ; \kappa) \prod_{l=1}^{n} \frac{\left[x_{i}-y_{l}+v+\lambda\right]}{\left[x_{i}-y_{l}+v\right]}+[\lambda] \sum_{k=1}^{n} A_{k}(y ; \lambda) \prod_{j=1}^{m} \frac{\left[y_{k}-x_{j}-v+\kappa\right]}{\left[y_{k}-x_{j}-v\right]}=0 .
$$

Also, when $[u]$ is a constant multiple of $\sin \left(\pi u / \omega_{1}\right)$ or $u$, we always have

$$
[\kappa] \sum_{i=1}^{m} A_{i}(x ; \kappa) \prod_{l=1}^{n} \frac{\left[x_{i}-y_{l}+v+\lambda\right]}{\left[x_{i}-y_{l}+v\right]}+[\lambda] \sum_{k=1}^{n} A_{k}(y ; \lambda) \prod_{j=1}^{m} \frac{\left[y_{k}-x_{j}-v+\kappa\right]}{\left[y_{k}-x_{j}-v\right]}=[m \kappa+n \lambda] .
$$

We now try to find a function $\Phi(x ; y)$ satisfying the system of first-order difference equations

$$
\begin{aligned}
& T_{x_{i}}^{\delta} \Phi(x ; y)=\prod_{l=1}^{n} \frac{\left[x_{i}-y_{l}+v+\lambda\right]}{\left[x_{i}-y_{l}+v\right]} \Phi(x ; y) \quad(i=1, \ldots, m), \\
& T_{y_{k}}^{\tau} \Phi(x ; y)=\prod_{j=1}^{m} \frac{\left[y_{k}-x_{j}-v+\kappa\right]}{\left[y_{k}-x_{j}-v\right]} \Phi(x ; y) \quad(k=1, \ldots, n),
\end{aligned}
$$

where $\tau$ stands for the unit scale of difference operators for $y$ variables. In order to fix the idea, assume that the balancing condition $m \kappa+n \lambda=0$ is satisfied. Then, any solution of this system should satisfy the functional equation

$$
[\kappa] \sum_{i=1}^{m} A_{i}(x ; \kappa) T_{x_{i}}^{\delta} \Phi(x ; y)+[\lambda] \sum_{k=1}^{n} A_{k}(y ; \lambda) T_{y_{k}}^{\tau} \Phi(x ; y)=0
$$

namely,

$$
[\kappa] D_{x}^{(\delta, \kappa)} \Phi(x ; y)+[\lambda] D_{y}^{(\tau, \lambda)} \Phi(x ; y)=0 .
$$


The compatibility condition for the system (3.4) of difference equations is given by

$$
\frac{\left[x_{i}-y_{k}-\tau+v+\lambda\right]\left[x_{i}-y_{k}+v\right]}{\left[x_{i}-y_{k}-\tau+v\right]\left[x_{i}-y_{k}+v+\lambda\right]}=\frac{\left[y_{k}-x_{i}-\delta-v+\kappa\right]\left[y_{k}-x_{i}-v\right]}{\left[y_{k}-x_{i}-\delta-v\right]\left[y_{k}-x_{i}-v+\kappa\right]}
$$

for $i=1, \ldots, m$ and $k=1, \ldots, n$. From this we see there are (at least) two cases where the difference equation (3.4) becomes compatible:

$$
\begin{array}{llll}
\text { (case 1) : } & \tau=-\delta, & \lambda=-\kappa, & v: \text { arbitrary, } \\
\text { (case 2) : } & \tau=\kappa, & \lambda=\delta, & v: \text { arbitrary. }
\end{array}
$$

In the first case, the difference equation to be solved is:

$$
\begin{aligned}
& T_{x_{i}}^{\delta} \Phi(x ; y)=\prod_{l=1}^{n} \frac{\left[x_{i}-y_{l}+v-\kappa\right]}{\left[x_{i}-y_{l}+v\right]} \Phi(x ; y) \quad(i=1, \ldots, m), \\
& T_{y_{k}}^{-\delta} \Phi(x ; y)=\prod_{j=1}^{m} \frac{\left[y_{k}-x_{j}-v+\kappa\right]}{\left[y_{k}-x_{j}-v\right]} \Phi(x ; y) \quad(k=1, \ldots, n) .
\end{aligned}
$$

This system is solved by

$$
\Phi_{A}^{-}(x ; y \mid \delta, \kappa)=\prod_{j=1}^{m} \prod_{l=1}^{n} \frac{G\left(x_{j}-y_{l}+v-\kappa \mid \delta\right)}{G\left(x_{j}-y_{l}+v \mid \delta\right)} .
$$

Hence we see that $\Phi_{A}^{-}(x ; y \mid \delta, \kappa)$ satisfies the functional equation

$$
D_{x}^{(\delta, \kappa)} \Phi_{A}^{-}(x ; y \mid \delta, \kappa)-D_{y}^{(-\delta,-\kappa)} \Phi_{A}^{-}(x ; y \mid \delta, \kappa)=0,
$$

under the balancing condition $(m-n) \kappa=0$, namely, $m=n$. By setting $\Phi_{A}(x ; y)=\Phi_{A}^{-}(x ;-y)$, we obtain

$$
D_{x}^{(\delta, \kappa)} \Phi_{A}(x ; y \mid \delta, \kappa)-D_{y}^{(\delta, \kappa)} \Phi_{A}(x ; y \mid \delta, \kappa)=0
$$

as in Theorem 2.1. The second case

$$
\begin{aligned}
T_{x_{i}}^{\delta} \Phi(x ; y) & =\prod_{l=1}^{n} \frac{\left[x_{i}-y_{l}+v+\delta\right]}{\left[x_{i}-y_{l}+v\right]} \Phi(x ; y) \quad(i=1, \ldots, m), \\
T_{y_{k}}^{\kappa} \Phi(x ; y) & =\prod_{j=1}^{m} \frac{\left[y_{k}-x_{j}-v+\kappa\right]}{\left[y_{k}-x_{j}-v\right]} \Phi(x ; y) \quad(k=1, \ldots, n)
\end{aligned}
$$

is solved by

$$
\Psi_{A}(x ; y)=\prod_{j=1}^{m} \prod_{l=1}^{n}\left[x_{j}-y_{l}+v\right] .
$$

Hence, under the balancing condition $m \kappa+n \delta=0$, we have

$$
[\kappa] D_{x}^{(\delta, \kappa)} \Psi_{A}(x ; y)+[\delta] D_{y}^{(\kappa, \delta)} \Psi_{A}(x ; y)=0 .
$$

This completes the proof of Theorem 2.1.

When $[u]$ is a constant multiple of $\sin \left(\pi u / \omega_{1}\right)$ or $u$, for any solution $\Phi(x ; y)$ of the system (3.4) of difference equations we have

$$
[\kappa] \sum_{i=1}^{m} A_{i}(x ; \kappa) T_{x_{i}}^{\delta} \Phi(x ; y)+[\lambda] \sum_{k=1}^{n} A_{k}(y ; \lambda) T_{y_{k}}^{\kappa} \Phi(x ; y)=[m \kappa+n \lambda] \Phi(x ; y),
$$


namely,

$$
[\kappa] D_{x}^{(\delta, \kappa)} \Phi(x ; y)+[\lambda] D_{y}^{(\tau, \lambda)} \Phi(x ; y)=[m \kappa+n \lambda] \Phi(x ; y)
$$

without imposing the balancing condition. This implies that the functions $\Phi_{A}(x ; y \mid \delta, \kappa)$ and $\Psi_{A}(x ; y)$ in these trigonometric and rational cases satisfy the functional equations

$$
D_{x}^{(\delta, \kappa)} \Phi_{A}(x ; y \mid \delta, \kappa)-D_{y}^{(\delta, \kappa)} \Phi_{A}(x ; y \mid \delta, \kappa)=\frac{[(m-n) \kappa]}{[\kappa]} \Phi_{A}(x ; y \mid \delta, \kappa)
$$

and

$$
[\kappa] D_{x}^{(\delta, \kappa)} \Psi_{A}(x ; y)+[\delta] D_{y}^{(\kappa, \delta)} \Psi_{A}(x ; y)=[m \kappa+n \delta] \Psi_{A}(x ; y) .
$$

respectively, as stated in Theorem 2.2 .

\subsection{Case of type $B C$}

In this $B C$ case, we assume that $[u]$ is a constant multiple of one of the following functions:

$$
u(\rho=1), \quad \sin \left(\pi u / \omega_{1}\right) \quad(\rho=2), \quad e\left(a u^{2}\right) \sigma(u ; \Omega) \quad(\rho=4) .
$$

In order to discuss difference operators of type $B C$, we consider the meromorphic function

$$
F(z)=\frac{\prod_{s=1}^{2 \rho}\left[z+\mu_{s}\right]}{\prod_{s=1}^{\rho}\left[z+\frac{1}{2}\left(\delta-\omega_{s}\right)\right]\left[z+\frac{1}{2}\left(\kappa-\omega_{s}\right)\right]} \prod_{j=1}^{m} \frac{\left[z \pm x_{j}+\kappa\right]}{\left[z \pm x_{j}\right]} \prod_{l=1}^{n} \frac{\left[z \pm y_{l}+v+\lambda\right]}{\left[z \pm y_{l}+v\right]} .
$$

By Proposition 3.1 it can be expanded as

$$
\begin{aligned}
{[c] F(z)=} & \sum_{i=1}^{m} \frac{\left[z-x_{i}+c\right]}{\left[z-x_{i}\right]} P_{i}^{+}+\sum_{i=1}^{m} \frac{\left[z+x_{i}+c\right]}{\left[z+x_{i}\right]} P_{i}^{-} \\
& +\sum_{k=1}^{n} \frac{\left[z-y_{k}+v+c\right]}{\left[z-y_{k}+v\right]} Q_{k}^{+}+\sum_{k=1}^{n} \frac{\left[z+y_{k}+v+c\right]}{\left[z+y_{k}+v\right]} Q_{k}^{-} \\
& +\sum_{r=1}^{\rho} \frac{\left[z+\frac{1}{2}\left(\delta-\omega_{r}\right)+c\right]}{\left[z+\frac{1}{2}\left(\delta-\omega_{r}\right)\right]} R_{r}+\sum_{r=1}^{\rho} \frac{\left[z+\frac{1}{2}\left(\kappa-\omega_{r}\right)+c\right]}{\left[z+\frac{1}{2}\left(\kappa-\omega_{r}\right)\right]} S_{r}
\end{aligned}
$$

into partial fractions, where

$$
c=2 m \kappa+2 n \lambda+\sum_{s=1}^{2 \rho} \mu_{s}-\frac{\rho}{2}(\delta+\kappa)+\sum_{s=1}^{\rho} \omega_{s}=2 m \kappa+2 n \lambda+c^{(\mu \mid \delta, \kappa)} .
$$

Also by Lemma 3.1, we see that expression

$$
\sum_{1 \leq i \leq m ; \epsilon= \pm} P_{i}^{\epsilon}+\sum_{1 \leq k \leq n ; \epsilon= \pm} Q_{k}^{\epsilon}+\sum_{1 \leq r \leq \rho} R_{r}+\sum_{1 \leq r \leq \rho} S_{r}
$$

reduces to 0 when the balancing condition $c=0$ is satisfied, or to $[c]$ when $[u]$ is trigonometric or rational. A remarkable fact is that, if the parameter $v$ is chosen appropriately, then the expansion coefficients of $F(z)$ are expressed in terms of the coefficients of Ruijsenaars operators of type $B C$. 
Proposition 3.2. When $v=\frac{1}{2}(\delta-\lambda)$, the function $F(z)$ defined by (3.5) is expressed as follows in terms of the coefficients of Ruijsenaars operators:

$$
\begin{aligned}
{[c] F(z)=} & \frac{[c] \prod_{s=1}^{2 \rho}\left[z+\mu_{s}\right]}{\prod_{s=1}^{\rho}\left[z+\frac{1}{2}\left(\delta-\omega_{s}\right)\right]\left[z+\frac{1}{2}\left(\kappa-\omega_{s}\right)\right]} \prod_{j=1}^{m} \frac{\left[z \pm x_{j}+\kappa\right]}{\left[z \pm x_{j}\right]} \prod_{l=1}^{n} \frac{\left[z \pm y_{l}+v+\lambda\right]}{\left[z \pm y_{l}+v\right]} \\
= & {[\kappa] \sum_{1 \leq i \leq m ; \epsilon= \pm} \frac{\left[z-\epsilon x_{i}+c\right]}{\left[z-\epsilon x_{i}\right]} A_{i}^{\epsilon}(x ; \mu \mid \delta, \kappa) \prod_{l=1}^{n} \frac{\left[\epsilon x_{i} \pm y_{l}+\frac{1}{2}(\delta+\lambda)\right]}{\left[\epsilon x_{i} \pm y_{l}+\frac{1}{2}(\delta-\lambda)\right]} } \\
& +[\kappa] \sum_{r=1}^{\rho} e\left(\frac{1}{2} c \eta_{r}\right) \frac{\left[z+\frac{1}{2}\left(\delta-\omega_{r}\right)+c\right]}{\left[z+\frac{1}{2}\left(\delta-\omega_{r}\right)\right]} A_{r}^{0}(x ; \mu \mid \delta, \kappa) \\
& +[\lambda] \sum_{1 \leq k \leq n ; \epsilon= \pm} \frac{\left[z-\epsilon y_{k}+v+c\right]}{\left[z-\epsilon y_{k}+v\right]} A_{k}^{\epsilon}(y ; \nu \mid \tau, \lambda) \prod_{j=1}^{m} \frac{\left[\epsilon y_{k} \pm x_{j}+\frac{1}{2}(\tau+\kappa)\right]}{\left[\epsilon y_{k} \pm x_{j}+\frac{1}{2}(\tau-\kappa)\right]} \\
& +[\lambda] \sum_{r=1}^{\rho} e\left(\frac{1}{2} c \eta_{r}\right) \frac{\left[z+\frac{1}{2}\left(\kappa-\omega_{r}\right)+c\right]}{\left[z+\frac{1}{2}\left(\kappa-\omega_{r}\right)\right]} A_{r}^{0}(y ; \nu \mid \tau, \lambda),
\end{aligned}
$$

where

$$
c=2 m \kappa+2 n \lambda+c^{(\mu \mid \delta, \kappa)}, \quad \tau=\kappa+\lambda-\delta, \quad \nu=\left(\mu_{1}-v, \ldots, \mu_{2 \rho}-v\right) .
$$

Proof. The expansion coefficients in (3.6) are determined from the residues at the corresponding poles. We first remark that

$$
P_{i}^{+}=\frac{[\kappa] \prod_{s=1}^{2 \rho}\left[x_{i}+\mu_{s}\right]}{\prod_{s=1}^{\rho}\left[x_{i}+\frac{1}{2}\left(\delta-\omega_{s}\right)\right]\left[x_{i}+\frac{1}{2}\left(\kappa-\omega_{s}\right)\right]} \frac{\left[2 x_{i}+\kappa\right]}{\left[2 x_{i}\right]} \prod_{j \neq i}^{m} \frac{\left[x_{i} \pm x_{j}+\kappa\right]}{\left[x_{i} \pm x_{j}\right]} \prod_{l=1}^{n} \frac{\left[x_{i} \pm y_{l}+v+\lambda\right]}{\left[x_{i} \pm y_{l}+v\right]} .
$$

Since

$$
\frac{\left[2 x_{i}+\kappa\right]}{\left[2 x_{i}\right]}=\prod_{s=1}^{\rho} \frac{\left[x_{i}+\frac{1}{2}\left(\kappa-\omega_{s}\right)\right]}{\left[x_{i}-\frac{1}{2} \omega_{s}\right]},
$$

we obtain

$$
\begin{aligned}
P_{i}^{+} & =\frac{[\kappa] \prod_{s=1}^{2 \rho}\left[x_{i}+\mu_{s}\right]}{\prod_{s=1}^{\rho}\left[x_{i}-\frac{1}{2} \omega_{s}\right]\left[x_{i}+\frac{1}{2}\left(\delta-\omega_{s}\right)\right]} \prod_{j \neq i}^{m} \frac{\left[x_{i} \pm x_{j}+\kappa\right]}{\left[x_{i} \pm x_{j}\right]} \prod_{l=1}^{n} \frac{\left[x_{i} \pm y_{l}+v+\lambda\right]}{\left[x_{i} \pm y_{l}+v\right]} \\
& =[\kappa] A_{i}^{+}(x ; \mu \mid \delta, \kappa) \prod_{l=1}^{n} \frac{\left[x_{i} \pm y_{l}+v+\lambda\right]}{\left[x_{i} \pm y_{l}+v\right]} .
\end{aligned}
$$

Note that $P_{i}^{-}$is obtained from $P_{i}^{+}$by replacing $x_{j}$ with $-x_{j}(j=1, \ldots, m)$. We next look at the coefficient $Q_{k}^{+}$:

$$
Q_{k}^{+}=\frac{[\lambda] \prod_{s=1}^{2 \rho}\left[y_{k}+\mu_{s}-v\right]}{\prod_{s=1}^{\rho}\left[y_{k}+\frac{1}{2}\left(\delta-\omega_{s}\right)-v\right]\left[y_{k}+\frac{1}{2}\left(\kappa-\omega_{s}\right)-v\right]} \frac{\left[2 y_{k}+\lambda\right]}{\left[2 y_{k}\right]}
$$




$$
\times \prod_{l \neq k} \frac{\left[y_{k} \pm y_{l}+\lambda\right]}{\left[y_{k} \pm y_{l}\right]} \prod_{j=1}^{m} \frac{\left[y_{k} \pm x_{j}+\kappa-v\right]}{\left[y_{k} \pm x_{j}-v\right]} .
$$

In view of

$$
\frac{\left[2 y_{k}+\lambda\right]}{\left[2 y_{k}\right]}=\prod_{s=1}^{\rho} \frac{\left[y_{k}+\frac{1}{2}\left(\lambda-\omega_{s}\right)\right]}{\left[y_{k}-\frac{1}{2} \omega_{s}\right]}
$$

we set $\frac{1}{2} \delta-v=\frac{1}{2} \lambda$, namely, $v=\frac{1}{2}(\delta-\lambda)$. Then we have

$$
\begin{aligned}
Q_{k}^{+} & =\frac{[\lambda] \prod_{s=1}^{2 \rho}\left[y_{k}+\mu_{s}-v\right]}{\prod_{s=1}^{\rho}\left[y_{k}-\frac{1}{2} \omega_{s}\right]\left[y_{k}+\frac{1}{2}\left(\tau-\omega_{s}\right)\right]} \prod_{l \neq k} \frac{\left[y_{k} \pm y_{l}+\lambda\right]}{\left[y_{k} \pm y_{l}\right]} \prod_{j=1}^{m} \frac{\left[y_{k} \pm x_{j}+\kappa-v\right]}{\left[y_{k} \pm x_{j}-v\right]} \\
& =[\lambda] A_{k}^{+}(y ; \nu \mid \tau, \lambda) \prod_{j=1}^{m} \frac{\left[y_{k} \pm x_{j}+\kappa-v\right]}{\left[y_{k} \pm x_{j}-v\right]}, \\
v & =\frac{1}{2}(\delta-\lambda), \quad \tau=\kappa+\lambda-\delta, \quad \nu=\left(\mu_{1}-v, \ldots, \mu_{2 \rho}-v\right) .
\end{aligned}
$$

The coefficient $Q_{k}^{-}$is obtained from $Q_{k}^{+}$by the sign change $y_{l} \rightarrow-y_{l}$. The coefficient $R_{r}$ is given by

$$
R_{r}=\frac{\prod_{s=1}^{2 \rho}\left[\frac{1}{2}\left(\omega_{r}-\delta\right)+\mu_{s}\right]}{\prod_{s \neq r}\left[\frac{1}{2} \omega_{r s}\right] \prod_{s=1}^{\rho}\left[\frac{1}{2} \omega_{r s}+\frac{1}{2}(\kappa-\delta)\right]} \prod_{j=1}^{m} \frac{\left[\frac{1}{2}\left(\omega_{r}-\delta\right) \pm x_{j}+\kappa\right]}{\left[\frac{1}{2}\left(\omega_{r}-\delta\right) \pm x_{j}\right]} \prod_{l=1}^{n} \frac{\left[\frac{1}{2}\left(\omega_{r}-\delta\right) \pm y_{l}+v+\lambda\right]}{\left[\frac{1}{2}\left(\omega_{r}-\delta\right) \pm y_{l}+v\right]},
$$

where $\omega_{r s}=\omega_{r}-\omega_{s}$. When $v=\frac{1}{2}(\delta-\lambda)$, we have

$$
\frac{\left[\frac{1}{2}\left(\omega_{r}-\delta\right) \pm y_{l}+v+\lambda\right]}{\left[\frac{1}{2}\left(\omega_{r}-\delta\right) \pm y_{l}+v\right]}=\frac{\left[\frac{1}{2}\left(\omega_{r}+\lambda\right) \pm y_{l}\right]}{\left[\frac{1}{2}\left(\omega_{r}-\lambda\right) \pm y_{l}\right]}=e\left(\lambda \eta_{r}\right)
$$

by the quasi-periodicity of $[u]$. In this way $y$ variables disappear from $R_{r}$ :

$$
R_{r}=\frac{e\left(n \lambda \eta_{r}\right) \prod_{s=1}^{2 \rho}\left[\frac{1}{2}\left(\omega_{r}-\delta\right)+\mu_{s}\right]}{\prod_{s \neq r}\left[\frac{1}{2} \omega_{r s}\right] \prod_{s=1}^{\rho}\left[\frac{1}{2} \omega_{r s}+\frac{1}{2}(\kappa-\delta)\right]} \prod_{j=1}^{m} \frac{\left[\frac{1}{2}\left(\omega_{r}-\delta\right) \pm x_{j}+\kappa\right]}{\left[\frac{1}{2}\left(\omega_{r}-\delta\right) \pm x_{j}\right]} .
$$

Note that the exponential factor $e\left(n \lambda \eta_{r}\right)$ is nontrivial only in the elliptic case. In any case, from $2 n \lambda=c-2 m \kappa-c^{(\mu \mid \delta, \kappa)}$ we have

$$
R_{r}=[\kappa] e\left(\frac{1}{2} c \eta_{r}\right) A_{r}^{0}(x ; \mu \mid \delta, \kappa) .
$$

Finally, when $v=\frac{1}{2}(\delta-\lambda)$ and $\tau=\kappa+\lambda-\delta$, we can rewrite $S_{r}$ as

$$
S_{r}=\frac{\prod_{s=1}^{2 \rho}\left[\frac{1}{2}\left(\omega_{r}-\kappa\right)+\mu_{s}\right]}{\prod_{s \neq r}\left[\frac{1}{2} \omega_{r s}\right] \prod_{s=1}^{\rho}\left[\frac{1}{2} \omega_{r s}+\frac{1}{2}(\delta-\kappa)\right]} \prod_{j=1}^{m} \frac{\left[\frac{1}{2}\left(\omega_{r}+\kappa\right) \pm x_{j}\right]}{\left[\frac{1}{2}\left(\omega_{r}-\kappa\right) \pm x_{j}\right]} \prod_{l=1}^{n} \frac{\left[\frac{1}{2}\left(\omega_{r}-\kappa\right) \pm y_{l}+v+\lambda\right]}{\left[\frac{1}{2}\left(\omega_{r}-\kappa\right) \pm y_{l}+v\right]}
$$




$$
=\frac{e\left(m \kappa \eta_{r}\right) \prod_{s=1}^{8}\left[\frac{1}{2}\left(\omega_{r}-\tau\right)+\mu_{s}-v\right]}{\prod_{s \neq r}\left[\frac{1}{2} \omega_{r s}\right] \prod_{s=1}^{4}\left[\frac{1}{2} \omega_{r s}+\frac{1}{2}(\lambda-\tau)\right]} \prod_{l=1}^{n} \frac{\left[\frac{1}{2}\left(\omega_{r}-\tau\right) \pm y_{l}+\lambda\right]}{\left[\frac{1}{2}\left(\omega_{r}-\tau\right) \pm y_{l}\right]} .
$$

Note that $x$ variables have disappeared again by the quasi-periodicity of $[u]$. Since $2 m \kappa=$ $c-2 n \lambda-c^{(\mu \mid \delta, \kappa)}$ and $c^{(\mu \mid \delta, \kappa)}=c^{(\nu \mid \tau, \lambda)}$, we have

$$
S_{r}=[\lambda] e\left(\frac{1}{2} c \eta_{r}\right) A_{r}^{0}(y ; \nu \mid \tau, \lambda) .
$$

This completes the proof of proposition.

In what follows we set $v=\frac{1}{2}(\delta-\lambda)$ and $\tau=\kappa+\lambda-\delta$. Then Proposition 3.2, combined with Lemma 3.1, implies

$$
\begin{aligned}
& {[\kappa] \sum_{1 \leq i \leq m ; \epsilon= \pm} A_{i}^{\epsilon}(x ; \mu \mid \delta, \kappa) \prod_{l=1}^{n} \frac{\left[\epsilon x_{i} \pm y_{l}+\frac{1}{2}(\delta+\lambda)\right]}{\left[\epsilon x_{i} \pm y_{l}+\frac{1}{2}(\delta-\lambda)\right]}+[\kappa] \sum_{s=1}^{\rho} A_{r}^{0}(x ; \mu \mid \delta, \kappa)} \\
& \quad+[\lambda] \sum_{1 \leq k \leq n ; \epsilon= \pm} A_{k}^{\epsilon}(y ; \nu \mid \tau, \lambda) \prod_{j=1}^{m} \frac{\left[\epsilon y_{k} \pm x_{j}+\frac{1}{2}(\tau+\kappa)\right]}{\left[\epsilon y_{k} \pm x_{j}+\frac{1}{2}(\tau-\kappa)\right]}+[\lambda] \sum_{r=1}^{\rho} A_{r}^{0}(y ; \nu \mid \tau, \lambda) \\
& =0, \quad\left(\operatorname{resp} .=\left[2 m \kappa+2 n \lambda+c^{(\mu \mid \delta, \kappa)}\right]\right),
\end{aligned}
$$

when the balancing condition $2 m \kappa+2 n \lambda+c^{(\mu \mid \delta, \kappa)}=0$ is satisfied (resp. when $[u]$ is trigonometric or rational.) Hence we have

Proposition 3.3. Suppose that the parameters $\delta, \kappa, \tau, \lambda$ satisfy the relation $\delta+\tau=\kappa+\lambda$, and define $\nu=\left(\nu_{1}, \ldots, \nu_{2 \rho}\right)$ by

$$
\nu_{s}=\mu_{s}-\frac{1}{2}(\delta-\lambda)=\mu_{s}+\frac{1}{2}(\tau-\kappa) \quad(s=1, \ldots, 2 \rho) .
$$

Let $\Phi(x ; y)$ any meromorphic function in the variables $x=\left(x_{1}, \ldots, x_{m}\right)$ and $y=\left(y_{1}, \ldots, y_{n}\right)$ satisfying the system of first-order difference equations

$$
\begin{aligned}
& T_{x_{i}}^{\delta} \Phi(x ; y)=\prod_{l=1}^{n} \frac{\left[x_{i} \pm y_{l}+\frac{1}{2}(\delta+\lambda)\right]}{\left[x_{i} \pm y_{l}+\frac{1}{2}(\delta-\lambda)\right]} \Phi(x ; y) \quad(i=1, \ldots, m), \\
& T_{y_{k}}^{\tau} \Phi(x ; y)=\prod_{j=1}^{m} \frac{\left[y_{k} \pm x_{j}+\frac{1}{2}(\tau+\kappa)\right]}{\left[y_{k} \pm x_{j}+\frac{1}{2}(\tau-\kappa)\right]} \Phi(x ; y) \quad(k=1, \ldots, n) .
\end{aligned}
$$

(1) If the balancing condition $2 m \kappa+2 n \lambda+c^{(\mu \mid \delta, \kappa)}=0$ is satisfied, then $\Phi(x ; y)$ satisfies the functional equation

$$
[\kappa] E_{x}^{(\mu \mid \delta, \kappa)} \Phi(x ; y)+[\lambda] E_{y}^{(\nu \mid \tau, \lambda)} \Phi(x ; y)=0 .
$$

(2) If $[u]$ is a constant multiple of $\sin \left(\pi u / \omega_{1}\right)$ or $u$, then $\Phi(x ; y)$ satisfies the functional equation

$$
[\kappa] E_{x}^{(\mu \mid \delta, \kappa)} \Phi(x ; y)+[\lambda] E_{y}^{(\nu \mid \tau, \lambda)} \Phi(x ; y)=\left[2 m \kappa+2 n \lambda+c^{(\mu \mid \delta, \kappa)}\right] \Phi(x ; y) .
$$

In fact there are essentially two cases where the system of first-order linear difference equations (3.7) become compatible:

$$
\begin{aligned}
& \text { (case 1): } \quad \tau=-\delta, \quad \lambda=-\kappa, \quad \nu_{s}=\mu_{s}-\frac{1}{2}(\delta+\kappa) \quad(s=1, \ldots, 2 \rho) \text {; } \\
& \text { (case 2): } \quad \tau=\kappa, \quad \lambda=\delta, \quad \nu_{s}=\mu_{s} \quad(s=1, \ldots, 2 \rho) \text {. }
\end{aligned}
$$


In the first case, the system (3.7) of difference equations to be solved is:

$$
\begin{aligned}
& T_{x_{i}}^{\delta} \Phi(x ; y)=\prod_{l=1}^{n} \frac{\left[x_{i} \pm y_{l}+\frac{1}{2}(\delta-\kappa)\right]}{\left[x_{i} \pm y_{l}+\frac{1}{2}(\delta+\kappa)\right]} \Phi(x ; y) \quad(i=1, \ldots, m), \\
& T_{y_{k}}^{\delta} \Phi(x ; y)=\prod_{j=1}^{m} \frac{\left[y_{k} \pm x_{j}+\frac{1}{2}(\delta-\kappa)\right]}{\left[y_{k} \pm x_{j}+\frac{1}{2}(\delta+\kappa)\right]} \Phi(x ; y) \quad(k=1, \ldots, n) .
\end{aligned}
$$

It is solved either by the function

$$
\Phi_{B C}(x ; y \mid \delta, \kappa)=\prod_{j=1}^{m} \prod_{l=1}^{n} \frac{G\left(x_{j} \pm y_{l}+\frac{1}{2}(\delta-\kappa) \mid \delta\right)}{G\left(x_{j} \pm y_{l}+\frac{1}{2}(\delta+\kappa) \mid \delta\right)},
$$

or by

$$
\Phi_{B C}(x ; y \mid \delta, \kappa)=\prod_{j=1}^{m} \prod_{l=1}^{n} \prod_{\epsilon_{1}, \epsilon_{2}= \pm} G\left(\epsilon_{1} x_{j}+\epsilon_{2} y_{l}+\frac{1}{2}(\delta-\kappa) \mid \delta\right) .
$$

Hence we see that, under the balancing condition $2(m-n) \kappa+c^{(\mu \mid \delta, \kappa)}=0, \Phi_{B C}(x ; y \mid \delta, \kappa)$ satisfies the functional equation

$$
E_{x}^{(\mu \mid \delta, \kappa)} \Phi_{B C}(x ; y \mid \delta, \kappa)-E_{y}^{(\nu \mid-\delta,-\kappa)} \Phi_{B C}(x ; y \mid \delta, \kappa)=0,
$$

for $\nu_{s}=\mu_{s}-\frac{1}{2}(\delta+\kappa)(s=1, \ldots, 2 \rho)$. By symmetry (2.12) with respect to the sign change of parameters, we obtain

$$
E_{x}^{(\mu \mid \delta, \kappa)} \Phi_{B C}(x ; y \mid \delta, \kappa)-E_{y}^{(\nu \mid \delta, \kappa)} \Phi_{B C}(x ; y \mid \delta, \kappa)=0,
$$

for $\nu_{s}=\frac{1}{2}(\delta+\kappa)-\mu_{s}(s=1, \ldots, 2 \rho)$. The second case

$$
\begin{array}{ll}
T_{x_{i}}^{\delta} \Phi(x ; y)=\prod_{l=1}^{n} \frac{\left[x_{i} \pm y_{l}+\delta\right]}{\left[x_{i} \pm y_{l}\right]} \Phi(x ; y) & (i=1, \ldots, m), \\
T_{y_{k}}^{\kappa} \Phi(x ; y)=\prod_{j=1}^{m} \frac{\left[y_{k} \pm x_{j}+\kappa\right]}{\left[y_{k} \pm x_{j}\right]} \Phi(x ; y) & (k=1, \ldots, n)
\end{array}
$$

is solved by

$$
\Psi_{B C}(x ; y)=\prod_{j=1}^{m} \prod_{l=1}^{n}\left[x_{j} \pm y_{l}\right]
$$

Hence, under the balancing condition $2 m \kappa+2 n \delta+c^{(\mu \mid \delta, \kappa)}=0$, this function satisfies the functional equation

$$
[\kappa] E_{x}^{(\mu \mid \delta, \kappa)} \Psi_{B C}(x ; y)+[\delta] E_{y}^{(\mu \mid \kappa, \delta)} \Psi_{B C}(x ; y)=0 .
$$

This completes the proof of Theorem 2.3. When $[u]$ is trigonometric or rational, Proposition $3.3,(2)$ implies that these functions $\Phi_{B C}(x ; y \mid \delta, \kappa)$ and $\Psi_{B C}(x ; y)$ satisfy the functional equations as stated in Theorem 2.4. 


\subsection{Difference operators of Koornwinder type}

In the rest of this section, we confine ourselves to the trigonometric and rational $B C$ cases and suppose that $[u]$ is a constant multiple of $\sin \left(\pi u / \omega_{1}\right)$ or $u$. We rewrite our results on kernel functions for these cases, in terms of difference operator

$$
D_{x}^{(\mu \mid \delta, \kappa)}=\sum_{i=1}^{m} A_{i}^{+}(x ; \mu \mid \delta, \kappa)\left(T_{x_{i}}^{\delta}-1\right)+\sum_{i=1}^{m} A_{i}^{-}(x ; \mu \mid \delta, \kappa)\left(T_{x_{i}}^{-\delta}-1\right)
$$

of Koornwinder type. We remark that this operator has symmetry

$$
D_{x}^{(\mu \mid-\delta,-\kappa)}=D_{x}^{(-\mu \mid \delta, \kappa)}
$$

with respect to the sign change, as in the case of $E_{x}^{(\mu \mid \delta, \kappa)}$. We show first that $D_{x}^{(\mu \mid \delta, \kappa)}$ differs from $E_{x}^{(\mu \mid \delta, \kappa)}$ only by an additive constant in the 0th order term.

\section{Lemma 3.2.}

(1) The constant function 1 is an eigenfunction of $E_{x}^{(\mu \mid \delta, \kappa)}$ :

$$
E_{x}^{(\mu \mid \delta, \kappa)}(1)=C^{(\mu \mid \delta, \kappa)}+\frac{1}{[\kappa]}\left(\left[2 m \kappa+c^{(\mu \mid \delta, \kappa)}\right]-\left[c^{(\mu \mid \delta, \kappa)}\right]\right) .
$$

(2) The two difference operators $D_{x}^{(\mu \mid \delta, \kappa)}$ and $E_{x}^{(\mu \mid \delta, \kappa)}$ are related as

$$
E_{x}^{(\mu \mid \delta, \kappa)}=D_{x}^{(\mu \mid \delta, \kappa)}+C^{(\mu \mid \delta, \kappa)}+\frac{1}{[\kappa]}\left(\left[2 m \kappa+c^{(\mu \mid \delta, \kappa)}\right]-\left[c^{(\mu \mid \delta, \kappa)}\right]\right) .
$$

Proof. Since $D_{x}^{(\mu \mid \kappa)}=E_{x}^{(\mu \mid \delta, \kappa)}-E_{x}^{(\mu \mid \delta, \kappa)}(1)$, statement (2) follows from statement (1). For the proof of (1), we make use of our Theorem 2.4. This theorem is valid even in the case where the dimension $m$ or $n$ reduces to zero. When $n=0$, Theorem 2.4, (1) implies

$$
[\kappa] E_{x}^{(\mu \mid \delta, \kappa)}(1)-[\kappa] C^{(\nu \mid \delta, \kappa)}=\left[2 m \kappa+c^{(\mu \mid \delta, \kappa)}\right],
$$

with $\nu_{s}=\frac{1}{2}(\delta+\kappa)-\mu_{s}(s=1, \ldots, 2 \rho)$, since $\Phi_{B C}(x ; y)$ in this case is the constant function 1 . Also from the case $m=n=0$ we have

$$
[\kappa] C^{(\mu \mid \delta, \kappa)}-[\kappa] C^{(\nu \mid \delta, \kappa)}=\left[c^{(\mu \mid \delta, \kappa)}\right] .
$$

Combining these two formulas we obtain

$$
[\kappa] E_{x}^{(\mu \mid \delta, \kappa)}(1)=[\kappa] C^{(\mu \mid \delta, \kappa)}+\left[2 m \kappa+c^{(\mu \mid \delta, \kappa)}\right]-\left[c^{(\mu \mid \delta, \kappa)}\right] .
$$

Let us rewrite the functional equations in Proposition 3.3 in terms of the operator $D_{x}^{(\mu \mid \delta, \kappa)}$. In the notation of Proposition 3.3, (2) we have

$$
[\kappa] E_{x}^{(\mu \mid \delta, \kappa)} \Phi(x ; y)+[\lambda] E_{y}^{(\nu \mid \tau, \lambda)} \Phi(x ; y)=\left[2 m \kappa+2 n \lambda+c^{(\mu \mid \delta, \kappa)}\right] \Phi(x ; y) .
$$

As special cases where $(m, n)=(m, 0),(0, n)$ and $(0,0)$, we have

$$
\begin{aligned}
& {[\kappa] E_{x}^{(\mu \mid \delta, \kappa)}(1)+[\lambda] C^{(\nu \mid \tau, \lambda)}=\left[2 m \kappa+c^{(\mu \mid \delta, \kappa)}\right],} \\
& {[\kappa] C^{(\mu \mid \delta, \kappa)}+[\lambda] E_{y}^{(\nu \mid \tau, \lambda)}(1)=\left[2 n \lambda+c^{(\mu \mid \delta, \kappa)}\right],} \\
& {[\kappa] C^{(\mu \mid \delta, \kappa)}+[\lambda] C^{(\nu \mid \tau, \lambda)}=\left[c^{(\mu \mid \delta, \kappa)}\right],}
\end{aligned}
$$


and hence

$$
\begin{aligned}
& -[\kappa] E_{x}^{(\mu \mid \delta, \kappa)}(1) \Phi(x ; y)-[\lambda] C^{(\nu \mid \tau, \lambda)} \Phi(x ; y)=-\left[2 m \kappa+c^{(\mu \mid \delta, \kappa)}\right] \Phi(x ; y), \\
& -[\kappa] C^{(\mu \mid \delta, \kappa)} \Phi(x ; y)-[\lambda] E_{y}^{(\nu \mid \tau, \lambda)}(1) \Phi(x ; y)=-\left[2 n \lambda+c^{(\mu \mid \delta, \kappa)}\right] \Phi(x ; y), \\
& {[\kappa] C^{(\mu \mid \delta, \kappa)} \Phi(x ; y)+[\lambda] C^{(\nu \mid \tau, \lambda)} \Phi(x ; y)=\left[c^{(\mu \mid \delta, \kappa)}\right] \Phi(x ; y),}
\end{aligned}
$$

By taking the sum of the four formulas in (3.9) and (3.10), we obtain

$$
\begin{aligned}
& {[\kappa] D_{x}^{(\mu \mid \delta, \kappa)} \Phi(x ; y)+[\lambda] D_{y}^{(\nu \mid \tau, \lambda)} \Phi(x ; y)=C \Phi(x ; y),} \\
& C=\left[2 m \kappa+2 n \lambda+c^{(\mu \mid \delta, \kappa)}\right]-\left[2 m \kappa+c^{(\mu \mid \delta, \kappa)}\right]-\left[2 n \lambda+c^{(\mu \mid \delta, \kappa)}\right]+\left[c^{(\mu \mid \delta, \kappa)}\right] .
\end{aligned}
$$

In the rational case, it is clear that $C=0$. In the trigonometric case, this constant $C$ can be factorized. In fact, if we choose $[u]=2 \sqrt{-1} \sin \left(\pi u / \omega_{1}\right)=e\left(u / 2 \omega_{1}\right)-e\left(-u / 2 \omega_{1}\right)$, we have a simple expression

$$
C=[m \kappa][n \lambda]\left[m \kappa+n \lambda+c^{(\mu \mid \delta, \kappa)}\right] .
$$

Hence, under the assumption of Proposition 3.3, (2), we have

$$
[\kappa] D_{x}^{(\mu \mid \delta, \kappa)} \Phi(x ; y)+[\lambda] D_{y}^{(\nu \mid \tau, \lambda)} \Phi(x ; y)=[m \kappa][n \lambda]\left[m \kappa+n \lambda+c^{(\mu \mid \delta, \kappa)}\right] \Phi(x ; y) \quad(\text { resp. }=0),
$$

when $[u]=e\left(u / 2 \omega_{1}\right)-e\left(-u / 2 \omega_{1}\right)$ (resp. when $\left.[u]=u\right)$. Applying this to the two cases of (3.8), one can easily derive functional equations as in Theorem 2.5.

\section{Kernel functions for $q$-difference operators}

In the trigonometric case, it is also important to consider $q$-difference operators passing to multiplicative variables. Assuming that $[u]=2 \sqrt{-1} \sin \left(\pi u / \omega_{1}\right)=e\left(u / 2 \omega_{1}\right)-e\left(-u / 2 \omega_{1}\right)$, we define by $z=e\left(u / \omega_{1}\right)$ the multiplicative variable associated with the additive variable $u$. When we write $[u]=z^{\frac{1}{2}}-z^{-\frac{1}{2}}=-z^{-\frac{1}{2}}(1-z)$, we regard the square root $z^{\frac{1}{2}}$ as a multiplicative notation for $e\left(u / 2 \omega_{1}\right)$. We set $q=e\left(\delta / \omega_{1}\right)$ and $t=e\left(\kappa / \omega_{1}\right)$, assuming that $\operatorname{Im}\left(\delta / \omega_{1}\right)>0$, namely, $|q|<1$.

\subsection{Kernel functions for Macdonald operators}

We introduce the multiplicative variables $z=\left(z_{1}, \ldots, z_{m}\right)$ and $w=\left(w_{1}, \ldots, w_{n}\right)$ corresponding to the additive variables $x=\left(x_{1}, \ldots, x_{m}\right)$ and $y=\left(y_{1}, \ldots, y_{n}\right)$, by $z_{i}=e\left(x_{i} / \omega_{1}\right)(i=1, \ldots, m)$ and $w_{k}=e\left(y_{k} / \omega_{1}\right)(k=1, \ldots, n)$, respectively.

In this convention of the trigonometric case, the Ruijsenaars difference operator $D_{x}^{(\delta, \kappa)}$ of type $A$ is a constant multiple of the $q$-difference operator of Macdonald:

$$
D_{x}^{(\delta, \kappa)}=t^{-\frac{1}{2}(m-1)} \mathcal{D}_{z}^{(q, t)}, \quad \mathcal{D}_{z}^{(q, t)}=\sum_{i=1}^{m} \prod_{1 \leq j \leq m ; j \neq i} \frac{t z_{i}-z_{j}}{z_{i}-z_{j}} T_{q, z_{i}},
$$

where $T_{q, z_{i}}$ denotes the $q$-shift operator with respect to $z_{i}$ :

$$
T_{q, z_{i}} f\left(z_{1}, \ldots, z_{m}\right)=f\left(z_{1}, \ldots, q z_{i}, \ldots, z_{m}\right) \quad(i=1, \ldots, m) .
$$

In what follows, we use the gamma function $G_{-}(u \mid \delta)$ of Section $2,(2.3)$. Then our kernel function $\Phi_{A}(x ; y \mid \delta, \kappa)$ with parameter $v=\kappa$ is expressed as follows in terms of multiplicative variables:

$$
\Phi_{A}(x ; y \mid \delta, \kappa)=e\left(\frac{m n \delta}{2 \omega_{1}}\left(\begin{array}{c}
\kappa / \delta \\
2
\end{array}\right)\right)\left(z_{1} \cdots z_{m}\right)^{\frac{n \kappa}{2 \delta}}\left(w_{1} \cdots w_{n}\right)^{\frac{m \kappa}{2 \delta}} \Pi(z ; w \mid q, t),
$$




$$
\Pi(z ; w \mid q, t)=\prod_{j=1}^{m} \prod_{l=1}^{n} \frac{\left(t z_{j} w_{l} ; q\right)_{\infty}}{\left(z_{j} w_{l} ; q\right)_{\infty}} .
$$

The functional equation of Theorem 2.2, (1) thus implies

$$
\mathcal{D}_{z}^{(q, t)} \Pi(z ; w \mid q, t)-t^{m-n} \mathcal{D}_{w}^{(q, t)} \Pi(z ; w \mid q, t)=\frac{1-t^{m-n}}{1-t} \Pi(z ; w \mid q, t),
$$

which can also be proved by the expansion formula

$$
\Pi(z ; w \mid q, t)=\sum_{l(\lambda) \leq \min \{m, n\}} b_{\lambda}(q, t) P_{\lambda}(z \mid q, t) P_{\lambda}(w \mid q, t)
$$

of Cauchy type for Macdonald polynomials [14]. (Formula (4.2) already implies that $\Pi(z ; w \mid q, t)$ has an expansion of this form, apart from the problem of determining the coefficients $b_{\lambda}(q, t)$.) On the other hand, kernel function $\Psi_{A}(x ; y)$ with parameter $v=0$ is expressed as

$$
\Psi_{A}(x ; y)=\left(z_{1} \cdots z_{m}\right)^{-\frac{n}{2}}\left(w_{1} \cdots w_{n}\right)^{-\frac{m}{2}} \prod_{j=1}^{m} \prod_{l=1}^{n}\left(z_{j}-w_{l}\right)
$$

Then the functional equation of Theorem 2.2, (2) implies

$$
\left((1-t) \mathcal{D}_{z}^{(q, t)}-(1-q) \mathcal{D}_{w}^{(t, q)}-\left(1-t^{m} q^{n}\right)\right) \prod_{j=1 l=1}^{m} \prod_{l=1}^{n}\left(z_{j}-w_{l}\right)=0
$$

This formula corresponds to the dual Cauchy formula

$$
\prod_{j=1 l=1}^{m} \prod^{n}\left(z_{j}-w_{l}\right)=\sum_{\lambda \subset\left(n^{m}\right)}(-1)^{\left|\lambda^{*}\right|} P_{\lambda}(z \mid q, t) P_{\lambda^{*}}(w \mid t, q),
$$

where $\lambda^{*}=\left(m-\lambda_{n}^{\prime}, \ldots, n-\lambda_{1}^{\prime}\right)$ is the partition representing the complement of $\lambda$ in the $m \times n$ rectangle.

These kernel functions for Macdonald operators have been applied to the studies of raising and lowering operators (Kirillov-Noumi [7, 8], Kajihara-Noumi [5]) and integral representation (Mimachi-Noumi [16], for instance). We also remark that, in this $A$ type case, a kernel function of Cauchy type for $q$-Dunkl operators has been constructed by Mimachi-Noumi [17].

\subsection{Kernel functions for Koornwinder operators}

We now consider the trigonometric $B C$ case. Instead of the additive parameters $\left(\mu_{1}, \mu_{2}, \mu_{3}, \mu_{4}\right)$ $(\rho=2)$, we use the multiplicative parameters

$$
(a, b, c, d)=\left(e\left(\mu_{1} / \omega_{1}\right), e\left(\mu_{2} / \omega_{1}\right), e\left(\mu_{3} / \omega_{1}\right), e\left(\mu_{4} / \omega_{1}\right)\right) .
$$

These four parameters are the Askey-Wilson parameters $(a, b, c, d)$ for the Koornwinder polynomials $P_{\lambda}(z ; a, b, c, d \mid q, t)$.

In this trigonometric $B C$ case, the difference operator

$$
D_{x}^{(\mu \mid \delta, \kappa)}=\sum_{i=1}^{m} A_{i}^{+}(x ; \mu \mid \delta, \kappa)\left(T_{x_{i}}^{\delta}-1\right)+\sum_{i=1}^{m} A_{i}^{-}(x ; \mu \mid \delta, \kappa)\left(T_{x_{i}}^{-\delta}-1\right),
$$


which we have discussed in Section 3.4, is a constant multiple of Koornwinder's q-difference operator [10]. Let us consider the Koornwinder operator

$$
\mathcal{D}_{z}^{(a, b, c, d \mid q, t)}=\sum_{i=1}^{m} \mathcal{A}_{i}^{+}(z)\left(T_{q, z_{i}}-1\right)+\sum_{i=1}^{m} \mathcal{A}_{i}^{-}(z)\left(T_{q, z_{i}}^{-1}-1\right)
$$

in the multiplicative variables, where the coefficients $\mathcal{A}_{i}^{+}(z)=\mathcal{A}_{i}^{+}(z ; a, b, c, d \mid q, t)$ are given by

$$
\mathcal{A}_{i}^{+}(z)=\frac{\left(1-a z_{i}\right)\left(1-b z_{i}\right)\left(1-c z_{i}\right)\left(1-d z_{i}\right)}{\left(a b c d q^{-1}\right)^{\frac{1}{2}} t^{m-1}\left(1-z_{i}^{2}\right)\left(1-q z_{i}^{2}\right)} \prod_{1 \leq j \leq m ; j \neq i} \frac{1-t z_{i} z_{j}^{ \pm 1}}{1-z_{i} z_{j}^{ \pm 1}}
$$

and $\mathcal{A}_{i}^{-}(z)=\mathcal{A}_{i}^{+}\left(z^{-1}\right)$ for $i=1, \ldots, m$. Then we have $D_{x}^{(\mu \mid \delta, \kappa)}=-\mathcal{D}_{z}^{(a, b, c, d \mid q, t)}$. Note that this operator $\mathcal{D}_{z}^{(a, b, c, d \mid q, t)}$ is renormalized by dividing the one used in [10] by the factor $\left(a b c d q^{-1}\right)^{\frac{1}{2}} t^{m-1}$. In what follows, we simply suppress the dependence on the parameters $(a, b, c, d \mid q, t)$ as $\mathcal{D}_{z}=$ $\mathcal{D}_{z}^{(a, b, c, d \mid q, t)}$, when we refer to operators or functions associated with these standard parameters.

In Section 2, we described two types of kernel functions (2.14) and (2.15) of Cauchy type. Also, depending on the choice of $G(u \mid \delta)$ we obtain several kernel functions for each type. From the gamma functions $G_{\mp}(u \mid \delta)$ of $(2.3)$, we obtain two kernel functions of type (2.14); in the multiplicative variables,

$$
\Phi_{0}(z ; w \mid q, t)=\left(z_{1} \cdots z_{m}\right)^{n \beta} \prod_{j=1}^{m} \prod_{l=1}^{n} \frac{\left(q^{\frac{1}{2}} t^{\frac{1}{2}} z_{j} w_{l}^{ \pm 1} ; q\right)_{\infty}}{\left(q^{\frac{1}{2}} t^{-\frac{1}{2}} z_{j} w_{l}^{ \pm 1} ; q\right)_{\infty}},
$$

and

$$
\Phi_{\infty}(z ; w \mid q, t)=\left(z_{1} \cdots z_{m}\right)^{-n \beta} \prod_{j=1}^{m} \prod_{l=1}^{n} \frac{\left(q^{\frac{1}{2}} t^{\frac{1}{2}} z_{j}^{-1} w_{l}^{ \pm 1} ; q\right)_{\infty}}{\left(q^{\frac{1}{2}} t^{-\frac{1}{2}} z_{j}^{-1} w_{l}^{ \pm 1} ; q\right)_{\infty}},
$$

respectively, where we put $\beta=\kappa / \delta$ so that $t=q^{\beta}$. Similarly, we obtain two kernel functions of type (2.15) from $G_{ \pm}(u \mid \delta)$ :

$$
\begin{aligned}
& \Phi_{+}(z ; w \mid q, t)=e\left(\frac{f(x ; y)}{\omega_{1} \delta}\right) \prod_{j=1}^{m} \prod_{l=1}^{n} \prod_{\epsilon_{1}, \epsilon_{2}= \pm}\left(q^{\frac{1}{2}} t^{\frac{1}{2}} z_{j}^{\epsilon_{1}} w_{l}^{\epsilon_{2}} ; q\right)_{\infty}, \\
& \Phi_{-}(z ; w \mid q, t)=e\left(-\frac{f(x ; y)}{\omega_{1} \delta}\right) \prod_{j=1}^{m} \prod_{l=1}^{n} \prod_{\epsilon_{1}, \epsilon_{2}= \pm}\left(q^{\frac{1}{2}} t^{-\frac{1}{2}} z_{j}^{\epsilon_{1}} w_{l}^{\epsilon_{2}} ; q\right)_{\infty}^{-1},
\end{aligned}
$$

where $f(x ; y)=n \sum_{j=1}^{m} x_{j}^{2}+m \sum_{l=1}^{n} y_{l}^{2}+\frac{m n}{4}\left(\kappa^{2}-\delta^{2}\right)$. Each of these four functions differs from the others by multiplicative factors which are $\delta$-periodic in all the $x$ variables and $y$ variables, It should be noted, however, that they have different analytic properties. In the following we denote simply by $\Phi(z ; w \mid q, t)$ one of these functions.

The kernel function $\Psi(z ; w)=\Psi_{B C}(x ; y)$ of dual Cauchy type is given by

$$
\Psi(z ; w)=\prod_{j=1}^{m} \prod_{l=1}^{n}\left(z_{j}+z_{j}^{-1}-w_{l}-w_{l}^{-1}\right)=\prod_{j=1 l=1}^{m} \prod_{l}^{n}\left(z_{j}-w_{l}\right)\left(1-z_{j}^{-1} w_{l}^{-1}\right),
$$

which is precisely the kernel function introduced by Mimachi [15].

For the passage from additive variables to multiplicative variables, we introduce the multiplicative notation for the function $[u]$ : For $z=e\left(u / \omega_{1}\right)$, we write $\langle z\rangle=[u]$. Namely, we set

$$
\langle z\rangle=z^{\frac{1}{2}}-z^{-\frac{1}{2}}=-z^{-\frac{1}{2}}(1-z), \quad z=e\left(u / \omega_{1}\right),
$$


with the square root $z^{\frac{1}{2}}$ regarded as the multiplicative notation for $e\left(u / 2 \omega_{1}\right)$. This function $\langle z\rangle$ is a natural object to be used in the case of $B C$ type, because of the symmetry $\left\langle z^{-1}\right\rangle=-\langle z\rangle$. In this notation, the coefficients $\mathcal{A}_{i}^{+}(z)$ of the Koornwinder operator $\mathcal{D}_{z}$ are expressed simply as

$$
\mathcal{A}_{i}^{+}(z)=\frac{\left\langle a z_{i}\right\rangle\left\langle b z_{i}\right\rangle\left\langle c z_{i}\right\rangle\left\langle d z_{i}\right\rangle}{\left\langle z_{i}^{2}\right\rangle\left\langle q z_{i}^{2}\right\rangle} \prod_{1 \leq j \leq m ; j \neq i} \frac{\left\langle t z_{i} / z_{j}\right\rangle\left\langle t z_{i} z_{j}\right\rangle}{\left\langle z_{i} / z_{j}\right\rangle\left\langle z_{i} z_{j}\right\rangle} \quad(i=1, \ldots, m) .
$$

It should be noted also that our parameter $c^{(\mu \mid \delta, \kappa)}=\sum_{s=1}^{4} \mu_{s}-(\delta+\kappa)+\omega_{1}$ passes to multiplicative variables as

$$
\left[u+c^{(\mu \mid \delta, \kappa)}\right]=-\left[u+\sum_{s=1}^{4} \mu_{s}-\delta-\kappa\right]=-\langle z a b c d / q t\rangle, \quad z=e\left(u / \omega_{1}\right),
$$

with a minus sign. Then, Theorem 2.5 can be restated as follows.

\section{Theorem 4.1.}

(1) The function $\Phi(z ; w \mid q, t)$ defined as above satisfies the functional equation

$$
\langle t\rangle \mathcal{D}_{z} \Phi(z ; w \mid q, t)-\langle t\rangle \widetilde{\mathcal{D}}_{w} \Phi(z ; w \mid q, t)=\left\langle t^{m}\right\rangle\left\langle t^{-n}\right\rangle\left\langle a b c d q^{-1} t^{m-n-1}\right\rangle \Phi(z ; w \mid q, t),
$$

where $\widetilde{\mathcal{D}}_{w}$ denotes the Koornwinder operator in $w$ variables with parameters $(a, b, c, d)$ replaced by $(\sqrt{q t} / a, \sqrt{q t} / b, \sqrt{q t} / c, \sqrt{q t} / d)$.

(2) The function $\Psi(z ; w)$ defined as (4.5) satisfies the functional equation

$$
\langle t\rangle \mathcal{D}_{z} \Psi(z ; w)+\langle q\rangle \widehat{\mathcal{D}}_{w} \Psi(z ; w)=\left\langle t^{m}\right\rangle\left\langle q^{n}\right\rangle\left\langle a b c d t^{m-1} q^{n-1}\right\rangle \Psi(z ; w),
$$

where $\widehat{D}_{w}$ denotes the Koornwinder operator in $w$ variables with parameters $(a, b, c, d \mid t, q)$.

Statement (2) of Theorem 4.1 recovers the key lemma of Mimachi [15, Lemma 3.2], from which he established the dual Cauchy formula

$$
\prod_{j=1 l=1}^{m} \prod^{n}\left(z_{j}+z_{j}^{-1}-w_{l}-w_{l}^{-1}\right)=\sum_{\lambda \subset\left(n^{m}\right)}(-1)^{\left|\lambda^{*}\right|} P_{\lambda}(z ; a, b, c, d \mid q, t) P_{\lambda^{*}}(w ; a, b, c, d \mid t, q)
$$

for Koornwinder polynomials, where the summation is taken over all partitions $\lambda=\left(\lambda_{1}, \ldots, \lambda_{m}\right)$ contained in the $m \times n$ rectangle, and $\lambda^{*}=\left(m-\lambda_{n}^{\prime}, \ldots, m-\lambda_{1}^{\prime}\right)$. By this formula, he also constructed an integral representation of Selberg type for Koornwinder polynomials attached to rectangles $\left(n^{m}\right)(n=0,1,2, \ldots)$. We expect that our kernel function $\Phi(z ; w \mid q, t)$ of Cauchy type could be applied as well to the study of eigenfunctions of the $q$-difference operators of Koornwinder. As a first step of such applications, in Section 5 we construct explicit formulas for Koornwinder polynomials attached to single columns and single rows.

\section{Application to Koornwinder polynomials}

In this section, we apply our results on the kernel functions for Koornwinder operators to the study of Koornwinder polynomials. In particular, we present new explicit formulas for Koornwinder polynomials attached to single columns and single rows.

To be more precise, we make use of the kernel functions to express Koornwinder polynomials $P_{\left(1^{r}\right)}(z ; a, b, c, d \mid q, t)(r=0,1, \ldots, m)$ and $P_{(l)}(z ; a, b, c, d \mid q, t)(l=0,1,2, \ldots)$ in terms of 
certain explicitly defined Laurent polynomials $E_{r}(z ; a \mid t)$ and $H_{l}(z ; a \mid q, t)$, respectively (Theorems 5.1 and 5.2). We remark that these Laurent polynomials $E_{r}(z ; a \mid t)$ are $H_{l}(z ; a \mid q, t)$ are in fact constant multiples of the $B C_{m}$ interpolation polynomials of Okounkov [18] attached to the partitions $\left(1^{r}\right)$ and $(l)$, respectively. (This fact will be proved in Appendix C.) Namely, Theorems 5.1 and 5.2 provide with two special cases of the binomial expansion of the Koornwinder polynomials in terms of $B C_{m}$ interpolation polynomials as is discussed in Okounkov [18] and Rains [19].

Once we establish the fact that $E_{r}(z ; a \mid t)$ and $H_{l}(z ; a \mid q, t)$ are interpolation polynomials, Theorems 5.1 and 5.2 can also be obtained from Okounkov's binomial formula [18], together with Rains' explicit evaluation of the binomial coefficients for the cases of $\left(1^{r}\right)$ and $(l)[19]^{1}$.

Before starting the discussion of Koornwinder polynomials, we introduce a notation

$$
\langle z ; w\rangle=\langle z w\rangle\langle z \mid w\rangle=z+z^{-1}-w-w^{-1}
$$

which corresponds to $[u \pm v]=[u+v][u-v]$ in additive variables. This expression, which appears frequently in the discussion of type $B C$, deserves a special attention. Note that

$$
\left\langle a^{-1} ; b\right\rangle=\left\langle a ; b^{-1}\right\rangle=\langle a ; b\rangle, \quad\langle b ; a\rangle=-\langle a ; b\rangle, \quad\langle a ; b\rangle+\langle b ; c\rangle=\langle a ; c\rangle,
$$

as clearly seen by the definition. Also, the Riemann relation for $[u]$ can be written as

$$
\langle z ; a\rangle\langle b ; c\rangle+\langle z ; b\rangle\langle c ; a\rangle+\langle z ; c\rangle\langle a ; b\rangle=0, \quad \frac{\langle z ; a\rangle}{\langle z ; b\rangle}-\frac{\langle w ; a\rangle}{\langle w ; b\rangle}=\frac{\langle z ; w\rangle\langle a ; b\rangle}{\langle z ; b\rangle\langle w ; b\rangle}
$$

\subsection{Koornwinder polynomials}

We briefly recall some basic facts about Koornwinder polynomials; for details, see Stokman [24] for example.

Let $\mathbb{K}=\mathbb{Q}\left(a^{\frac{1}{2}}, b^{\frac{1}{2}}, c^{\frac{1}{2}}, d^{\frac{1}{2}}, q^{\frac{1}{2}}, t^{\frac{1}{2}}\right)$ be the field of rational functions in indeterminates, representing the square roots of the parameters $a, b, c, d, q$, , with coefficients in $\mathbb{Q}$, and $\mathbb{K}\left[z^{ \pm 1}\right]=\mathbb{K}\left[z_{1}^{ \pm 1}, \ldots, z_{m}^{ \pm 1}\right]$ the ring of Laurent polynomials in $m$ variables $z=\left(z_{1}, \ldots, z_{m}\right)$ with coefficients in $\mathbb{K}$. Then the Weyl group $W=\{ \pm 1\}^{m} \rtimes \mathfrak{S}_{m}$ of type $B C$ (hyperoctahedral group) acts naturally on $\mathbb{K}\left[z^{ \pm 1}\right]$ through the permutation of indices for the $z$ variables and the individual inversion of variables $z_{i}(i=1, \ldots, m)$. The Koornwinder polynomial $P_{\lambda}(z)=P_{\lambda}(z ; a, b, c, d \mid q, t)$ attached to a partition $\lambda=\left(\lambda_{1}, \ldots, \lambda_{m}\right)$ is then characterized as a unique $W$-invariant Laurent polynomial in $\mathbb{K}\left[z^{ \pm 1}\right]$ satisfying the following two conditions:

(1) $P_{\lambda}(z)$ is expressed in terms of orbit sums $m_{\mu}(z)=\sum_{\nu \in W . \mu} z^{\nu}$ as

$$
P_{\lambda}(z)=m_{\lambda}(z)+\sum_{\mu<\lambda} c_{\lambda, \mu} m_{\mu}(z) \quad\left(c_{\lambda, \mu} \in \mathbb{K}\right)
$$

where $\leq$ stands for the dominance ordering of partitions.

(2) $P_{\lambda}(z)$ is an eigenfunction of Koornwinder's $q$-difference operator $\mathcal{D}_{z}$ :

$$
\mathcal{D}_{z} P_{\lambda}(z)=d_{\lambda} P_{\lambda}(z) \quad \text { for some } \quad d_{\lambda} \in \mathbb{K}
$$

\footnotetext{
${ }^{1}$ The authors thank Professor Eric Rains for pointing out this connection with the interpolation polynomials and the binomial formula.
} 
These polynomials $P_{\lambda}(z)$, indexed by partitions $\lambda$, form a $\mathbb{K}$-basis of the ring of $W$-invariants $\mathbb{K}\left[z^{ \pm 1}\right]^{W}$. Also, the eigenvalues $d_{\lambda}$ are given by

$$
d_{\lambda}=\sum_{i=1}^{m}\left\langle\alpha t^{m-i} q^{\lambda_{i}} ; \alpha t^{m-i}\right\rangle=\sum_{i=1}^{m}\left(\alpha t^{m-i} q^{\lambda_{i}}+\alpha^{-1} t^{-m+i} q^{-\lambda_{i}}\right)-\sum_{i=1}^{m}\left(\alpha t^{m-i}+\alpha^{-1} t^{-m+i}\right),
$$

in our notation $\langle z ; w\rangle=z+z^{-1}-w-w^{-1}$, where $\alpha=\left(a b c d q^{-1}\right)^{\frac{1}{2}}$. (Note that $P_{0}(z)=1$, and $d_{0}=0$.)

We give below new explicit formulas for Koornwinder polynomials $P_{\left(1^{r}\right)}(z)$ attached to single columns $\left(1^{r}\right)(r=0,1, \ldots, m)$, and $P_{(r)}(z)$ attached to single rows $(r=0,1,2 \ldots)$. As we already mentioned, our explicit formulas provide with the two special cases of Okounkov's binomial expansion of the Koornwinder polynomials in terms of $B C_{m}$ interpolation polynomials. We also remark that, in the cases of type $B, C, D$, some conjectures have been proposed by Lassalle [11] on explicit formulas for Macdonald polynomials attached to single rows. The relationship between his conjectures and our approach will be discussed in a separate paper.

In order to formulate our results, we define a set of $W$-invariant Laurent polynomials $E_{r}(z ; a)$ with reference point a $(r=0,1, \ldots, m)$ by

$$
E_{r}(z ; a \mid t)=\sum_{1 \leq i_{1}<\cdots<i_{r} \leq m}\left\langle z_{i_{1}} ; t^{i_{1}-1} a\right\rangle\left\langle z_{i_{2}} ; t^{i_{2}-2} a\right\rangle \cdots\left\langle z_{i_{r}} ; t^{i_{r}-r} a\right\rangle .
$$

As we will see below, these Laurent polynomials are $W$-invariant in spite of their appearance, and they can be considered as a variation of the orbit sums $m_{\left(1^{r}\right)}(z)(r=0,1, \ldots, m)$ attached to the fundamental weights. In fact, these Laurent polynomials $E_{r}(z ; a \mid t)(r=0,1, \ldots, m)$ are essentially the $B C_{m}$ interpolation polynomials of Okounkov attached to single columns $\left(1^{r}\right.$ ) (for a proof, see Appendix C). We remark that these polynomials had appeared already in the work of van Diejen [3] in relation to the eigenvalues of his commuting family $q$-difference operators for this $B C_{m}$ case. They are also used effectively by a recent work of Aomoto-Ito [1] in their study of Jackson integrals of type $B C$.

Theorem 5.1. The Koornwinder polynomials $P_{\left(1^{r}\right)}(z ; a, b, c, d \mid q, t)$ attached to columns $\left(1^{r}\right)$ $(r=0,1, \ldots, m)$ are expressed as follows in terms of $E_{s}(z ; a \mid t)(s=0,1, \ldots, m)$ :

$$
P_{\left(1^{r}\right)}(z ; a, b, c, d \mid q, t)=\sum_{l=0}^{r} \frac{\left\langle t^{m-r+1}, t^{m-r} a b, t^{m-r} a c, t^{m-r} a d\right\rangle_{t, l}}{\left\langle t, t^{2(m-r)} a b c d\right\rangle_{t, l}} E_{r-l}(z ; a \mid t),
$$

where $\langle a\rangle_{t, l}=\langle a\rangle\langle t a\rangle \cdots\left\langle t^{l-1} a\right\rangle$, and $\left\langle a_{1}, \ldots, a_{r}\right\rangle_{t, l}=\left\langle a_{1}\right\rangle_{t, l} \cdots\left\langle a_{r}\right\rangle_{t, l}$.

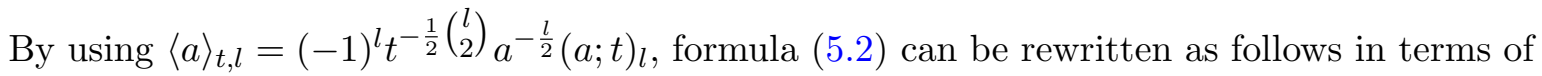
ordinary $t$-shifted factorials of [4]:

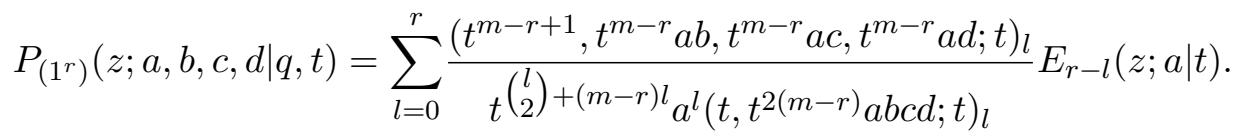

For the description of Koornwinder polynomials attached to single rows, we introduce a sequence of $W$-invariant Laurent polynomials $H_{l}(z ; a \mid q, t)(l=0,1,2, \ldots)$ as follows:

$$
\begin{aligned}
& H_{l}(z ; a \mid q, t) \\
& =\sum_{\nu_{1}+\cdots+\nu_{m}=l} \frac{\langle t\rangle_{q, \nu_{1}} \cdots\langle t\rangle_{q, \nu_{m}}}{\langle q\rangle_{q, \nu_{1}} \cdots\langle q\rangle_{q, \nu_{m}}}\left\langle z_{1} ; a\right\rangle_{q, \nu_{1}}\left\langle z_{2} ; t q^{\nu_{1}} a\right\rangle_{q, \nu_{2}} \cdots\left\langle z_{m} ; t^{m-1} q^{\nu_{1}+\cdots+\nu_{m-1}} a\right\rangle_{q, \nu_{m}},
\end{aligned}
$$


where

$$
\langle z ; a\rangle_{q, l}=\langle z ; a\rangle\langle z ; q a\rangle \cdots\left\langle z ; q^{l-1} a\right\rangle=(-1)^{l} q^{-\left(\begin{array}{l}
l \\
2
\end{array}\right)^{-l}\left(a z, a z^{-1} ; q\right)_{l} .}
$$

Note that $\langle z ; a\rangle_{q, l}$ is a monic Laurent polynomial in $z$ of degree $l$. (The $W$-invariance of $H_{l}(z ; a \mid q, t)$ will be proved in Lemma 5.4 below.) These Laurent polynomials $H_{l}(z ; a \mid q, t)$ can be regarded as a $B C_{m}$ analogue of the $A_{m-1}$ Macdonald polynomials attached to single rows:

$$
\frac{(t ; q)_{r}}{(q ; q)_{r}} P_{(r)}^{A}(z)=Q_{(r)}^{A}(z)=\sum_{\nu_{1}+\cdots+\nu_{m}=r} \frac{(t ; q)_{\nu_{1}} \cdots(t ; q)_{\nu_{m}}}{(q ; q)_{\nu_{1}} \cdots(q ; q)_{\nu_{m}}} z_{1}^{\nu_{1}} \cdots z_{m}^{\nu_{m}} .
$$

Also, they are special cases of $B C_{m}$ interpolation polynomials attached to single rows $(l)$ (see Appendix C).

Theorem 5.2. The Koornwinder polynomials $P_{(r)}(z ; a, b, c, d \mid q, t)$ attached to rows $(r)(r=$ $0,1,2, \ldots)$ are expressed as follows in terms of $H_{l}(z ; a \mid q, t)(l=0,1,2, \ldots)$ :

$$
\begin{aligned}
\frac{\langle t\rangle_{q, r}}{\langle q\rangle_{q, r}} P_{(r)}(z ; a, b, c, d \mid q, t)= & \frac{\left\langle t^{m}, t^{m-1} a b, t^{m-1} a c, t^{m-1} a d\right\rangle_{q, r}}{\left\langle q, t^{2(m-1)} a b c d q^{r-1}\right\rangle_{q, r}} \\
& \times \sum_{l=0}^{r} \frac{(-1)^{l}\left\langle q^{-r}, t^{2(m-1)} a b c d q^{r-1}\right\rangle_{q, l}}{\left\langle t^{m}, t^{m-1} a b, t^{m-1} a c, t^{m-1} a d\right\rangle_{q, l}} H_{l}(z ; a \mid q, t) .
\end{aligned}
$$

Let us denote by $p_{r}(z ; a, b, c, d \mid q)$ the Koornwinder polynomial $P_{(r)}(z ; a, b, c, d \mid q, t)$ in the one variable case $(r=0,1,2, \ldots)$. Note that, when $m=1, H_{l}(z ; a \mid q, t)$ reduces to $\langle t\rangle_{q, l}\langle z ; a\rangle_{q, l} /\langle q\rangle_{q, l}$. Hence Theorem 5.2 for $m=1$ implies

$$
\begin{aligned}
p_{r}(z ; a, b, c, d \mid q) & =\frac{\langle a b, a c, a d\rangle_{q, r}}{\left\langle a b c d q^{r-1}\right\rangle_{q, r}} \sum_{l=0}^{r} \frac{(-1)^{l}\left\langle q^{-r}, a b c d q^{r-1}\right\rangle_{q, l}}{\langle q, a b, a c, a d\rangle_{q, l}}\langle z ; a\rangle_{q, l} \\
& =\frac{(a b, a c, a d ; q)_{r}}{a^{r}\left(a b c d q^{r-1} ; q\right)_{r}}{ }_{4} \phi_{3}\left[\begin{array}{c}
q^{-r}, a b c d q^{r-1}, a z, a / z \\
a b, a c, a d
\end{array}{ }^{q}, q\right]
\end{aligned}
$$

which recovers the well-known ${ }_{4} \phi_{3}$ representation of the (monic) Askey-Wilson polynomials.

We prove these Theorems 5.1 and 5.2 in Subsections 5.2 and 5.3, by means of the kernel functions of dual Cauchy type, and of Cauchy type, respectively.

\subsection{Case of a single column}

We first explain some properties of the elementary Laurent polynomials $E_{r}(z ; a \mid t)(r=0,1$, $\ldots, m)$.

Lemma 5.1. The Laurent polynomials $E_{r}(z ; a \mid t)(r=0,1, \ldots, m)$ are characterized as the expansion coefficients in

$$
\prod_{j=1}^{m}\left\langle w ; z_{j}\right\rangle=\sum_{r=0}^{m}(-1)^{r} E_{r}(z ; a \mid t)\langle w ; a\rangle_{t, m-r}
$$

where $\langle w ; a\rangle_{t, l}=\langle w ; a\rangle\langle w ; t a\rangle \cdots\left\langle w ; t^{l-1} a\right\rangle$. In particular, $E_{r}(z ; a \mid t)$ is $W$-invariant for each $r=0,1, \ldots, m$.

Proof. Since the uniqueness of expansion in terms of $\langle w ; a\rangle_{t, r}(r=0,1, \ldots, m)$ is obvious, we show the validity of the expansion formula above. Note that $E_{r}(z ; a \mid t)$ can be expressed as

$$
E_{r}(z ; a \mid t)=\sum_{|I|=r i \in I} \prod_{i \in I}\left\langle z_{i} ; t^{\left|I_{<i}^{\mathrm{c}}\right|} a\right\rangle, \quad I_{<i}^{\mathrm{c}}=\{j \in\{1, \ldots, m\} \backslash I \mid j<i\} .
$$


Hence we have the recurrence formula

$$
E_{r}(z ; a \mid t)=\left\langle z_{1} ; a\right\rangle E_{r-1}\left(z^{\prime} ; a \mid t\right)+E_{r}\left(z^{\prime} ; t a \mid t\right), \quad z^{\prime}=\left(z_{2}, \ldots, z_{m}\right) .
$$

By using this recurrence, one can inductively prove the expansion formula of lemma. In fact, by $\left\langle w ; z_{1}\right\rangle=\langle w ; a\rangle-\left\langle z_{1} ; a\right\rangle$, we compute

$$
\begin{aligned}
\prod_{j=1}^{m}\left\langle w ; z_{j}\right\rangle= & \langle w ; a\rangle \prod_{i=2}^{m}\left\langle w ; z_{i}\right\rangle-\left\langle z_{1} ; a\right\rangle \prod_{i=2}^{m}\left\langle w ; z_{i}\right\rangle \\
= & \langle w ; a\rangle \sum_{r=0}^{m-1}(-1)^{r} E_{r}\left(z^{\prime} ; t a \mid t\right)\langle w ; t a\rangle_{t, m-1-r} \\
& -\left\langle z_{1} ; a\right\rangle \sum_{s=0}^{m-1}(-1)^{s} E_{s}\left(z^{\prime} ; a \mid t\right)\langle w ; a\rangle_{t, m-1-s} \\
= & \sum_{r=0}^{m}(-1)^{r}\left(E_{r}\left(z^{\prime} ; t a \mid t\right)+\left\langle z_{1} ; a\right\rangle E_{r-1}\left(z^{\prime} ; a \mid t\right)\right)\langle w ; a\rangle_{t, m-r} \\
= & \sum_{r=0}^{m}(-1)^{r} E_{r}(z ; a \mid t)\langle w ; a\rangle_{t, m-r} .
\end{aligned}
$$

We now proceed to the proof of Theorem 5.1. Mimachi's dual Cauchy formula for Koornwinder polynomials can be written as

$$
\prod_{j=1 l=1}^{m}\left\langle\prod_{l}^{n}\left\langle w_{l} ; z_{j}\right\rangle=\sum_{\lambda \subset\left(n^{m}\right)}(-1)^{|\lambda|} P_{\lambda}(z ; a, b, c, d \mid q, t) P_{\lambda^{*}}(w ; a, b, c, d \mid t, q) .\right.
$$

When $n=1$, this formula implies

$$
\prod_{j=1}^{m}\left\langle w ; z_{j}\right\rangle=\sum_{r=0}^{m}(-1)^{r} P_{\left(1^{r}\right)}(z \mid q, t) p_{m-r}(w \mid t)
$$

where we have omitted the parameters $(a, b, c, d)$. Namely, the Koornwinder polynomials attached to single columns are determined as expansion coefficients of the kernel function for $n=1$ in terms of the monic Askey-Wilson polynomials $p_{l}(w \mid t)=p_{l}(w ; a, b, c, d \mid t)$ with base $t$. On the other hand, we already have the expansion formula

$$
\prod_{j=1}^{m}\left\langle w ; z_{j}\right\rangle=\sum_{l=0}^{m}(-1)^{l} E_{l}(z ; a \mid t)\langle w ; a\rangle_{t, m-l} .
$$

Recalling that

$$
\begin{aligned}
p_{l}(w \mid t) & =\frac{\langle a b, a c, a d\rangle_{t, l}}{\left\langle a b c d t^{l-1}\right\rangle_{t, l}} \sum_{r=0}^{l}(-1)^{r} \frac{\left\langle t^{-l}, a b c d t^{l-1}\right\rangle_{t, r}}{\langle t, a b, a c, a d\rangle_{t, r}}\langle w ; a\rangle_{t, r} \\
& =\sum_{r=0}^{l} \frac{\left\langle t^{r+1}, t^{r} a b, t^{r} a c, t^{r} a d\right\rangle_{t, l-r}}{\left\langle t, a b c d t^{l+r-1}\right\rangle_{t, l-r}}\langle w ; a\rangle_{t, r} \quad(l=0,1,2, \ldots)
\end{aligned}
$$

we consider to express $\langle w ; a\rangle_{t, l}$ in terms of Askey-Wilson polynomials $p_{r}(w \mid t)$.

Lemma 5.2. For each $l=0,1, \ldots$, one has

$$
\langle w ; a\rangle_{t, l}=\sum_{r=0}^{l}(-1)^{l-r} \frac{\left\langle t^{r+1}, t^{r} a b, t^{r} a c, t^{r} a d\right\rangle_{t, l-r}}{\left\langle t, a b c d t^{2 r}\right\rangle_{t, l-r}} p_{r}(w \mid t) \quad(l=0,1,2, \ldots) .
$$


We omit the proof of this lemma, since it can be derived as a special case of the connection formula for Askey-Wilson polynomials with different parameters (see [4]). Note that, if we set $d=t^{1-l} / a$ in (5.8), then $p_{l}\left(w ; a, b, c, t^{1-l} / a \mid t\right)=\langle w ; a\rangle_{t, l}$.

By substituting (5.9) into (5.7), we obtain

$$
\prod_{j=1}^{m}\left\langle w ; z_{j}\right\rangle=\sum_{0 \leq l \leq r \leq m}(-1)^{r} \frac{\left\langle t^{m-r+1}, t^{m-r} a b, t^{m-r} a c, t^{m-r} a d\right\rangle_{r-l}}{\left\langle t, a b c d t^{2(m-r)}\right\rangle_{t, r-l}} E_{l}(z ; a \mid t) p_{m-r}(w \mid t) .
$$

Comparing this formula with (5.6) we obtain

$$
P_{\left(1^{r}\right)}(z \mid q, t)=\sum_{l=0}^{r} \frac{\left\langle t^{m-r+1}, t^{m-r} a b, t^{m-r} a c, t^{m-r} a d\right\rangle_{r-l}}{\left\langle t, a b c d t^{2(m-r)}\right\rangle_{t, r-l}} E_{l}(z ; a \mid t),
$$

as desired.

\subsection{Case of a single row}

Recall that the kernel function of Cauchy type

$$
\Phi(z ; w \mid q, t)=\left(z_{1} \cdots z_{m}\right)^{n \beta} \prod_{j=1}^{m} \prod_{l=1}^{n} \frac{\left(q^{\frac{1}{2}} t^{\frac{1}{2}} z_{j} w_{l}^{ \pm 1} ; q\right)_{\infty}}{\left(q^{\frac{1}{2}} t^{-\frac{1}{2}} z_{j} w_{l}^{ \pm 1} ; q\right)_{\infty}}
$$

defined in (4.4), satisfies the difference equation

$$
\langle t\rangle \mathcal{D}_{z} \Phi(z ; w \mid q, t)-\langle t\rangle \widetilde{\mathcal{D}}_{w} \Phi(z ; w \mid q, t)=\left\langle t^{m}\right\rangle\left\langle t^{-n}\right\rangle\left\langle a b c d q^{-1} t^{m-n-1}\right\rangle \Phi(z ; w \mid q, t),
$$

where $\widetilde{\mathcal{D}}_{w}$ denotes the Koornwinder operator in $w$ variables with parameters $(a, b, c, d)$ replaced by $(\sqrt{q t} / a, \sqrt{q t} / b, \sqrt{q t} / c, \sqrt{q t} / d \mid q, t)$. We set hereafter

$$
\widetilde{a}=\sqrt{q t} / a, \quad \widetilde{b}=\sqrt{q t} / b, \quad \widetilde{c}=\sqrt{q t} / c, \quad \widetilde{d}=\sqrt{q t} / d .
$$

Also, for any Laurent polynomial $f(z) \in \mathbb{K}\left[z^{ \pm 1}\right]$, we denote by $\widetilde{f}(z) \in \mathbb{K}\left[z^{ \pm 1}\right]$ the Laurent polynomial obtained from $f(z)$ by replacing the parameters $(a, b, c, d)$ with $(\widetilde{a}, \widetilde{b}, \widetilde{c}, \widetilde{d})$.

Let us consider the special case where $t=q^{-k}(k=0,1,2, \ldots)$. Then the kernel function $\Phi\left(z ; w \mid q, q^{-k}\right)$ reduces to a Laurent polynomial in $(z, w)$ :

$$
\Phi\left(z ; w \mid q, q^{-k}\right)=\left(z_{1} \cdots z_{m}\right)^{-k n} \prod_{j=1 l=1}^{m} \prod^{n}\left(q^{\frac{1}{2}(1-k)} z_{j} w_{l}^{ \pm 1} ; q\right)_{k}=(-1)^{k m n} \prod_{j=1 l=1}^{m} \prod_{l=1}^{n}\left\langle w_{l} ; q^{\frac{1}{2}(1-k)} z_{j}\right\rangle_{q, k} .
$$

Ignoring the sign factor, we set

$$
\Phi_{-k}(z ; w)=\prod_{j=1}^{m} \prod_{l=1}^{n}\left\langle w_{l} ; q^{\frac{1}{2}(1-k)} z_{j}\right\rangle_{q, k} .
$$

Note that

$$
\left\langle w ; q^{\frac{1}{2}(1-k)} z\right\rangle_{q, k}=\left\langle w ; q^{\frac{1}{2}(-k+1)} z\right\rangle\left\langle w ; q^{\frac{1}{2}(-k+2)} z\right\rangle \cdots\left\langle w ; q^{\frac{1}{2}(k-1)} z\right\rangle
$$

is invariant under the inversion $z \rightarrow z^{-1}$. In what follows, we analyze the case where $n=1$ and $t=q^{-k}(k=0,1,2, \ldots)$. In this case, the kernel function

$$
\Phi_{-k}(z ; w)=\prod_{j=1}^{m}\left\langle w ; q^{\frac{1}{2}(1-k)} z_{j}\right\rangle_{k}
$$


is a symmetric Laurent polynomial in $w$ of degree $\mathrm{km}$. Also, this kernel function satisfies the functional equation

$$
\mathcal{D}_{z} \Phi_{-k}(z ; w)-\widetilde{\mathcal{D}}_{w} \Phi_{-k}(z ; w)=-\left\langle t^{m}\right\rangle\left\langle a b c d q^{-1} t^{m-2}\right\rangle \Phi_{-k}(z ; w) .
$$

With $\alpha=\left(a b c d q^{-1}\right)^{\frac{1}{2}}$, this formula can be written as

$$
\mathcal{D}_{z} \Phi_{-k}(z ; w)=\left(\widetilde{\mathcal{D}}_{w}-\left\langle\alpha t^{m-1} ; \alpha t^{-1}\right\rangle\right) \Phi_{-k}(z ; w) .
$$

Noting that $\Phi_{-k}(z ; w)$ is a symmetric Laurent polynomial, we expand this kernel in terms of the monic Askey-Wilson polynomials $\widetilde{p}_{l}(w \mid q)=p_{l}(w ; \widetilde{a}, \widetilde{b}, \widetilde{c}, \widetilde{d} \mid q)$ in $w$ with the twisted parameters, so that

$$
\Phi_{-k}(z ; w)=\prod_{j=1}^{m}\left\langle w ; q^{\frac{1}{2}(1-k)} z_{j}\right\rangle_{k}=\sum_{l=0}^{k m} G_{l}(z) \widetilde{p}_{k m-l}(w \mid q) .
$$

The Laurent polynomials $G_{l}(z)(0 \leq l \leq k m)$ are uniquely determined by this expansion, and hence $W$-invariant.

Lemma 5.3. When $t=q^{-k}(k=0,1,2, \ldots)$, the $W$-invariant Laurent polynomials $G_{l}(z)$ $(0 \leq l \leq k m)$ defined as above are eigenfunctions of $\mathcal{D}_{z}$ :

$$
\mathcal{D}_{z} G_{l}(z)=\left\langle\alpha t^{m-1} q^{l} ; \alpha t^{m-1}\right\rangle G_{l}(z) \quad(0 \leq l \leq k m) .
$$

Proof. Note that

$$
\mathcal{D}_{w} p_{k m-l}(w \mid q)=\left\langle\alpha q^{k m-l} ; \alpha\right\rangle p_{k m-l}(w \mid q)=\left\langle\alpha t^{-m} q^{-l} ; \alpha\right\rangle p_{k m-l}(w \mid q),
$$

where $\alpha=\left(a b c d q^{-1}\right)^{\frac{1}{2}}$. Hence, by $\widetilde{\alpha}=t q^{\frac{1}{2}}(a b c d)^{-\frac{1}{2}}=t / \alpha$, we obtain

$$
\widetilde{\mathcal{D}}_{w} \widetilde{p}_{k m-l}(w \mid q)=\left\langle t^{1-m} q^{-l} / \alpha ; t / \alpha\right\rangle \widetilde{p}_{k m-l}(w \mid q)=\left\langle\alpha t^{m-1} q^{l} ; \alpha t^{-1}\right\rangle \widetilde{p}_{k m-l}(w \mid q) .
$$

In view of (5.10), we compute

$$
\begin{aligned}
\left(\widetilde{\mathcal{D}}_{w}-\left\langle\alpha t^{m-1} ; \alpha t^{-1}\right\rangle\right) \widetilde{p}_{k m-l}(w \mid q) & =\left(\left\langle\alpha t^{m-1} q^{l} ; \alpha t^{-1}\right\rangle-\left\langle\alpha t^{m-1} ; \alpha t^{-1}\right\rangle\right) \widetilde{p}_{k m-l}(w \mid q) \\
& =\left\langle\alpha t^{m-1} q^{l} ; \alpha t^{m-1}\right\rangle \widetilde{p}_{k m-l}(w \mid q) .
\end{aligned}
$$

Hence, (5.10) implies

$$
\begin{aligned}
\sum_{l=0}^{k m} \mathcal{D}_{z} G_{l}(z) \widetilde{p}_{k m-l}(w \mid q) & =\sum_{l=0}^{k m} G_{l}(z)\left(\widetilde{\mathcal{D}}_{w}-\left\langle\alpha t^{m-1} ; \alpha t^{-1}\right\rangle\right) \widetilde{p}_{k m-l}(w \mid q) \\
& =\sum_{l=0}^{k m}\left\langle\alpha t^{m-1} q^{l} ; \alpha t^{m-1}\right\rangle G_{l}(z) \widetilde{p}_{k m-l}(w \mid q) .
\end{aligned}
$$

Namely,

$$
\mathcal{D}_{z} G_{l}(z)=\left\langle\alpha t^{m-1} q^{l} ; \alpha t^{m-1}\right\rangle G_{l}(z) \quad(l=0,1, \ldots, k m) .
$$

As we have seen above, each $G_{l}(z)(l=0,1, \ldots, k m)$ is an eigenfunction of $\mathcal{D}_{z}$ with precisely the same eigenvalue as the one for the Koornwinder polynomial $P_{(l)}(z)$ attached to the single row of length $l$. At this moment, however, we cannot conclude this $G_{l}(z)$ is indeed a constant 
multiple of the Koornwinder polynomial $P_{(l)}(z)$ specialized to the case $t=q^{-k}$. This is because different partitions $\lambda$ may give the same eigenvalue

$$
d_{\lambda}=\sum_{i=1}^{m}\left\langle\alpha t^{m-i} q^{\lambda_{i}} ; \alpha t^{m-i}\right\rangle
$$

under this specialization. This point will be discussed later after we determine an explicit formula for $G_{l}(z)$.

Since we already know the relationship between the Askey-Wilson polynomials $p_{l}(w \mid q)$ and the Laurent polynomials $\langle w ; a\rangle_{q, l}$, we consider to expand the kernel function $\Phi_{-k}(z ; w)$ in terms of $\langle w ; a\rangle_{q, l}$.

\section{Lemma 5.4.}

(1) When $t=q^{-k}(k=0,1,2, \ldots)$, the kernel function $\Phi_{-k}(z ; w)$ has the following expansion formula:

$$
\Phi_{-k}(z ; w)=\prod_{j=1}^{m}\left\langle w ; q^{\frac{1}{2}(1-k)} z_{j}\right\rangle_{q, k}=\sum_{l=0}^{k m} H_{l}(z)\langle w ; \sqrt{q t} / a\rangle_{q, k m-l}
$$

where $H_{l}(z)$ stands for $H_{l}(z ; a \mid q, t)$ defined as (5.3) with $t=q^{-k}$ for $l=0,1, \ldots, k m$.

(2) The Laurent polynomials $H_{l}(z ; a \mid q, t)(l=0,1,2, \ldots)$ are $W$-invariant.

Proof. Statement (2) follows from the expansion formula (5.11) of statement (1). Since $\Phi_{-k}(z ; w)$ is $W$-invariant in the $z$ variables, formula (5.11) implies that $H_{l}\left(z ; a \mid q, q^{-k}\right)$ is $W$ invariant for $k \geq l / m$. Hence we see that $H_{l}(z ; a \mid q, t)$ itself is $W$-invariant as a Laurent polynomial in $\mathbb{K}\left[z^{ \pm}\right]$for each $l=0,1,2, \ldots$.

In the following proof of statement (1), we omit the base $q$, and write $\langle w ; a\rangle_{l}=\langle w ; a\rangle_{q, l}$. We first present a connection formula for the Laurent polynomials $\langle w ; a\rangle_{l}$ and $\langle w ; b\rangle_{l}$ with different reference point $a, b$ :

$$
\langle w ; b\rangle_{l}=\sum_{r=0}^{l}(-1)^{r}\left[\begin{array}{l}
l \\
r
\end{array}\right]\left\langle q^{l-r} a b, b / a\right\rangle_{r}\langle w ; a\rangle_{l-r}, \quad\left[\begin{array}{l}
l \\
r
\end{array}\right]=(-1)^{r} \frac{\left\langle q^{-l}\right\rangle_{r}}{\langle q\rangle_{r}},
$$

which is equivalent to the $q$-Saalschütz sum [4]

$$
\frac{(b w, b / w ; q)_{l}}{(b a, b / a ; q)_{l}}={ }_{3} \phi_{2}\left[\begin{array}{c}
q^{-l}, a w, a / w \\
a b, q^{1-l} a / b
\end{array} ; q, q\right] .
$$

Let us rewrite the formula above in the form

$$
\langle w ; b\rangle_{l}=\sum_{r=0}^{l}(-1)^{r}\left[\begin{array}{l}
l \\
r
\end{array}\right]\left\langle q^{\frac{1}{2}(l-1)} b ; q^{\frac{1}{2}(1-l)} / a\right\rangle_{r}\langle w ; a\rangle_{l-r} .
$$

Hence,

$$
\left\langle w ; q^{\frac{1}{2}(1-\mu)} z\right\rangle_{\mu}=\sum_{\nu=0}^{\mu}(-1)^{\nu}\left[\begin{array}{l}
\mu \\
\nu
\end{array}\right]\left\langle z ; q^{\frac{1}{2}(1-\mu)} / a\right\rangle_{\nu}\langle w ; a\rangle_{\mu-\nu} .
$$

This implies

$$
\left\langle w ; q^{\frac{1}{2}\left(1-\mu_{1}\right)} z_{1}\right\rangle_{\mu_{1}}\left\langle w ; q^{\frac{1}{2}\left(1-\mu_{2}\right)} z_{2}\right\rangle_{\mu_{2}}
$$




$$
=\sum_{\nu_{1}=0}^{\mu_{1}}(-1)^{\nu_{1}}\left[\begin{array}{l}
\mu_{1} \\
\nu_{1}
\end{array}\right]\left\langle z_{1} ; q^{\frac{1}{2}\left(1-\mu_{1}\right)} / a\right\rangle_{\nu_{1}}\langle w ; a\rangle_{\mu_{1}-\nu_{1}}\left\langle w ; q^{\frac{1}{2}\left(1-\mu_{2}\right)} z_{2}\right\rangle_{\mu_{2}} .
$$

Then in each term, we expand $\left\langle w ; q^{\frac{1}{2}\left(1-\mu_{2}\right)} z_{2}\right\rangle_{\mu_{2}}$ in terms of $\left\langle w ; q^{\mu_{1}-\nu_{1}} a\right\rangle_{\mu_{2}-\nu_{2}}\left(0 \leq \nu_{2} \leq \mu_{2}\right)$ to get

$$
\begin{aligned}
& \left\langle w ; q^{\frac{1}{2}\left(1-\mu_{1}\right)} z_{1}\right\rangle_{\mu_{1}}\left\langle w ; q^{\frac{1}{2}\left(1-\mu_{2}\right)} z_{2}\right\rangle_{\mu_{2}} \\
& =\sum_{\nu_{1}=0}^{\mu_{1}} \sum_{\nu_{2}=0}^{\mu_{2}}(-1)^{\nu_{1}+\nu_{2}}\left[\begin{array}{c}
\mu_{1} \\
\nu_{1}
\end{array}\right]\left[\begin{array}{c}
\mu_{2} \\
\nu_{2}
\end{array}\right]\left\langle z_{1} ; q^{\frac{1}{2}\left(1-\mu_{1}\right)} / a\right\rangle_{\nu_{1}} \\
& \quad \times\left\langle z_{2} ; q^{\frac{1}{2}\left(1-\mu_{2}\right)-\left(\mu_{1}-\nu_{1}\right)} / a\right\rangle_{\nu_{2}}\langle w ; a\rangle_{\mu_{1}+\mu_{2}-\nu_{1}-\nu_{2}} .
\end{aligned}
$$

By repeating this procedure, we finally obtain

$$
\prod_{j=1}^{m}\left\langle w ; q^{\frac{1}{2}\left(1-\mu_{j}\right)} z_{j}\right\rangle_{\mu_{j}}=\sum_{\nu}(-1)^{|\nu|} \prod_{j=1}^{m}\left[\begin{array}{c}
\mu_{j} \\
\nu_{j}
\end{array}\right]\left\langle z_{j} ; q^{\frac{1}{2}\left(1-\mu_{j}\right)-\sum_{i<j}\left(\mu_{i}-\nu_{i}\right)} / a\right\rangle_{\nu_{j}}\langle w ; a\rangle_{|\mu|-|\nu|}
$$

for any $\mu=\left(\mu_{1}, \ldots, \mu_{m}\right)$, where the sum is taken over all multi-indices $\nu=\left(\nu_{1}, \ldots, \nu_{m}\right)$ such that $\nu_{i} \leq \mu_{i}(i=1, \ldots, m)$. As a special case of this formula where $\mu_{1}=\cdots=\mu_{m}=k$, $|\mu|=k m$, we get the expansion formula

$$
\Phi_{-k}(z ; w)=\prod_{j=1}^{m}\left\langle w ; q^{\frac{1}{2}(1-k)} z_{j}\right\rangle_{k}=\sum_{l=0}^{k m} \widetilde{H}_{l}(z)\langle w ; a\rangle_{k m-l}
$$

for $\Phi_{-k}(z ; w)$. Here the coefficients are determined as

$$
\begin{aligned}
\widetilde{H}_{l}(z) & =\sum_{|\nu|=l}(-1)^{|\nu|} \prod_{j=1}^{m}\left[\begin{array}{c}
k \\
\nu_{j}
\end{array}\right]\left\langle z_{j} ; q^{\frac{1}{2}(1-k)-k(j-1)+\sum_{i<j} \nu_{i}} / a\right\rangle_{\nu_{j}} \\
& =\sum_{\nu_{1}+\cdots+\nu_{m}=l} \prod_{j=1}^{m} \frac{\langle t\rangle_{\nu_{j}}}{\langle q\rangle_{\nu_{j}}}\left\langle z_{j} ; t^{j-1} q^{\sum_{i<j} \nu_{i}} \sqrt{q t} / a\right\rangle_{\nu_{j}}
\end{aligned}
$$

with $t=q^{-k}$. Replacing the parameters $a$ by $\widetilde{a}=\sqrt{q t} / a$, we obtain

$$
\Phi_{-k}(z ; w)=\prod_{j=1}^{m}\left\langle w ; q^{\frac{1}{2}(1-k)} z_{j}\right\rangle_{k}=\sum_{l=0}^{k m} H_{l}(z)\langle w ; \sqrt{q t} / a\rangle_{k m-l}
$$

where

$$
H_{l}(z)=\sum_{\nu_{1}+\cdots+\nu_{m}=l j=1} \prod_{j}^{m} \frac{\langle t\rangle_{\nu_{j}}}{\langle q\rangle_{\nu_{j}}}\left\langle z_{j} ; t^{j-1} q^{\sum_{i<j} \nu_{i}} a\right\rangle_{\nu_{j}} \quad\left(t=q^{-k}\right) .
$$

We now have two expansions of the kernel function $\Phi_{-k}(z ; w)$ :

$$
\Phi_{-k}(z ; w)=\sum_{r=0}^{k m} G_{r}(z) \widetilde{p}_{k m-r}(w \mid q)=\sum_{l=0}^{k m} H_{l}(z)\langle w ; \sqrt{q t} / a\rangle_{q, k m-l} .
$$

Also, from Lemma 5.2 we see

$$
\langle w ; a\rangle_{q, l}=\sum_{r=0}^{l}(-1)^{l-r} \frac{\left\langle q^{r+1}, q^{r} a b, q^{r} a c, q^{r} a d\right\rangle_{q, l-r}}{\left\langle q, a b c d q^{2 r}\right\rangle_{q, l-r}} p_{r}(w \mid q),
$$


and hence,

$$
\langle w ; \sqrt{q t} / a\rangle_{q, l}=\sum_{r=0}^{l}(-1)^{l-r} \frac{\left\langle q^{r+1}, t q^{r+1} / a b, t q^{r+1} / a c, t q^{r+1} / a d\right\rangle_{q, l-r}}{\left\langle q, t^{2} q^{2(r+1)} / a b c d\right\rangle_{q, l-r}} \widetilde{p}_{r}(w \mid q)
$$

for $l=0,1,2, \ldots$. Substituting this into (5.12), we obtain an expression of $G_{r}(z)$ in term of $H_{l}(z)$ as follows:

$$
\begin{aligned}
G_{r}(z) & =\sum_{l=0}^{r}(-1)^{r-l} \frac{\left\langle q^{k m-r+1}, t q^{k m-r+1} / a b, t q^{k m-r+1} / a c, t q^{k m-r+1} / a d\right\rangle_{q, r-l}}{\left\langle q, t^{2} q^{2(k m-r+1)} / a b c d\right\rangle_{q, r-l}} H_{l}(z) \\
& =\sum_{l=0}^{r}(-1)^{r-l} \frac{\left\langle q^{1-r} t^{-m}, q^{1-r} t^{1-m} / a b, q^{1-r} t^{1-m} / a c, q^{1-r} t^{1-m} / a d\right\rangle_{q, r-l}}{\left\langle q, q^{2(1-r)} t^{2(1-m)} / a b c d\right\rangle_{q, r-l}} H_{l}(z) \\
& =\frac{\left\langle t^{m}, t^{m-1} a b, t^{m-1} a c, t^{m-1} a d\right\rangle_{q, r}}{\left\langle q, t^{2(m-1)} a b c d q^{r-1}\right\rangle_{q, r}} \sum_{l=0}^{r} \frac{(-1)^{l}\left\langle q^{-r}, t^{2(m-1)} a b c d q^{r-1}\right\rangle_{q, l}}{\left\langle t^{m}, t^{m-1} a b, t^{m-1} a c, t^{m-1} a d\right\rangle_{q, l}} H_{l}(z) .
\end{aligned}
$$

From the expression obtained above, it is clear that

$$
H_{l}(z)=\frac{\langle t\rangle_{q, l}}{\langle q\rangle_{q, l}} m_{(l)}(z)+\text { terms lower than }(l) \text { with respect to } \leq,
$$

and

$$
G_{r}(z)=\frac{\langle t\rangle_{q, r}}{\langle q\rangle_{q, r}} m_{(r)}(z)+\text { terms lower than }(r) \text { with respect to } \leq
$$

Note that $\langle t\rangle_{q, r}=\left\langle q^{-k}\right\rangle_{q, r} \neq 0$ for $0 \leq r \leq k$. Also, we already know that each $G_{r}(z)(r=$ $0,1, \ldots, k m)$ satisfies the difference equation

$$
\mathcal{D}_{z} G_{r}(z)=\left\langle\alpha t^{m-1} q^{r} ; \alpha t^{m-1}\right\rangle G_{r}(z)
$$

Suppose in general that a partition $\lambda=\left(\lambda_{1}, \ldots, \lambda_{m}\right)$ satisfies the condition $\lambda_{i}-\lambda_{i+1} \leq k$ $(i=1, \ldots, m-1)$. Since

$$
\lambda_{1}+k \leq \lambda_{2}+2 k \leq \cdots \leq \lambda_{m}+k m
$$

in this case, it turns out that the eigenvalue $d_{\mu}$ for any partition $\mu<\lambda$ is distinct from $d_{\lambda}$ when the square roots of $a, b, c, d, q$ are regarded as indeterminates. For such a partition $\lambda$, the Koornwinder polynomial $P_{\lambda}(z)=P_{\lambda}(z ; a, b, c, d \mid q, t)$ can be specialized to $t=q^{-k}$, and any eigenfunction having the nontrivial leading term $m_{\lambda}(z)$ must be a constant multiple of $P_{\lambda}\left(z ; a, b, c, d \mid q, q^{-k}\right)$. This implies that, for each $r$ with $0 \leq r \leq k, G_{r}(z)$ is a constant multiple of $P_{(r)}(z)$ specialized to $t=q^{-k}$ :

$$
G_{r}(z)=\left.\frac{\langle t\rangle_{q, r}}{\langle q\rangle_{q, r}} P_{(r)}(z)\right|_{t=q^{-k}} \quad(0 \leq r \leq k) .
$$

For each $r=0,1,2, \ldots$, consider the Laurent polynomial in $\mathbb{K}\left[z^{ \pm 1}\right]$ defined by right-hand side of the explicit formula (5.4) of Theorem 5.2. Then the both sides of (5.4) are regular at $t=q^{-k}$ $(k \geq r)$, and they coincide with each other for $t=q^{-k}(k=r, r+1, \ldots)$. Hence the both sides must be identical as rational functions in $t^{\frac{1}{2}}$. This completes the proof of Theorem 5.2. 


\section{A Remarks on higher order difference operators}

In the case of type $A$, an explicit commuting family of higher order difference operators, denoted below by $D_{r, x}^{(\delta, \kappa)}(r=1, \ldots, m)$, including $D_{x}^{(\delta, \kappa)}$ as a first member, has been constructed by Ruijsenaars [20]. He also proved in [23] that, when $m=n$, the kernel function of Cauchy type, corresponding to our $\Phi_{A}(x ; y \mid \delta, \kappa)$, intertwines the whole commuting families of difference operators in $x$ variable and $y$ variables. (For the comparison of our $\Phi_{A}(x ; y \mid \delta, \kappa)$ with the type $A$ kernel functions of Ruijsenaars [23], see Appendix B.1.)

Fix any nonzero entire function $[x]$ satisfying the Riemann relation as in Section 2. We consider a sequence of difference operators $D_{r, x}^{(\delta, \kappa)}(r=1, \ldots, m)$ defined by

$$
D_{r, x}^{(\delta, \kappa)}=\sum_{I \subset\{1, \ldots, m\} ;|I|=r} \prod_{i \in I, j \notin I} \frac{\left[x_{i}-x_{j}+\kappa\right]}{\left[x_{i}-x_{j}\right]} \prod_{i \in I} T_{x_{i}}^{\delta} .
$$

Then, from the result of [20] and its degenerate cases, it follows that these operators $D_{r, x}^{(\delta, \kappa)}$ $(r=1, \ldots, m)$ commute with each other. In this setting the same kernel function

$$
\Phi_{A}(x ; y \mid \delta, \kappa)=\prod_{j, l=1}^{m} \frac{G\left(x_{j}+y_{l}+v-\kappa \mid \delta\right)}{G\left(x_{j}+y_{l}+v \mid \delta\right)}
$$

as in (2.5) for the case $m=n$ satisfies the difference equation

$$
D_{r, x}^{(\delta, \kappa)} \Phi_{A}(x ; y \mid \delta, \kappa)=D_{r, y}^{(\delta, \kappa)} \Phi_{A}(x ; y \mid \delta, \kappa)
$$

for all $r=1, \ldots, m$. This functional equation is in fact equivalent to

$$
\begin{aligned}
\sum_{|I|=r} & \prod_{i \in I ; j \notin I} \frac{\left[x_{i}-x_{j}+\kappa\right]}{\left[x_{i}-x_{j}\right]} \prod_{i \in I ; 1 \leq l \leq m} \frac{\left[x_{i}+y_{l}+v-\kappa\right]}{\left[x_{i}+y_{l}+v\right]} \\
& =\sum_{|K|=r} \prod_{k \in K ; l \notin K} \frac{\left[y_{k}-y_{l}+\kappa\right]}{\left[y_{k}-y_{l}\right]} \prod_{k \in K ; 1 \leq j \leq m} \frac{\left[y_{k}+x_{j}+v-\kappa\right]}{\left[y_{k}+x_{j}+v\right]},
\end{aligned}
$$

which is precisely the key identity of Kajihara-Noumi [6, Theorem 1.3], that was derived from the determinantal formula of Frobenius.

In the trigonometric case, it is convenient to consider the generating function

$$
\mathcal{D}_{z}^{(q, t)}(u)=\sum_{r=0}^{m}(-u)^{r} \mathcal{D}_{r, z}^{(q, t)}, \quad \mathcal{D}_{r, z}^{(q, t)}=t^{\left(\begin{array}{c}
r \\
2
\end{array}\right)} \sum_{|I|=r} \prod_{i \in I ; j \notin I} \frac{t z_{i}-z_{j}}{z_{i}-z_{j}} \prod_{i \in I} T_{q, z_{i}}
$$

of the Macdonald $q$-difference operators, passing to the multiplicative variables. Then from the eigenfunction expansion (4.3), it follows that the kernel function $\Pi(z ; w \mid q, t)$ of (4.1) for the variables $z=\left(z_{1}, \ldots, z_{m}\right)$ and $w=\left(w_{1}, \ldots, w_{n}\right)$ satisfies the functional equation

$$
\left(t^{m} u ; t\right)_{\infty} \mathcal{D}_{z}^{(q, t)}\left(t^{n} u\right) \Pi(z ; w \mid q, t)=\left(t^{n} u ; t\right)_{\infty} \mathcal{D}_{w}^{(q, t)}\left(t^{m} u\right) \Pi(z ; w \mid q, t) .
$$

It would be an important problem to find an elliptic extension of this formula for the case $m \neq n$.

As for type $B C$, a commuting family of higher order difference operators for $E_{x}^{(\mu \mid \delta, \kappa)}$ has been constructed explicitly by van Diejen $[2,3]$ in the trigonometric case, and inductively by Komori-Hikami [9] in the elliptic case. We expect that our kernel function $\Phi_{B C}(x ; y \mid \delta, \kappa)$ should intertwine the whole commuting families of higher order difference operators in $x$ variables and $y$ variables (at least under the balancing condition in the elliptic case), similarly to the $A$ type case. 


\section{B Comparison with [23]}

As we already mentioned, in his series of works [23] Ruijsenaars has constructed kernel function for elliptic difference operators of type $\left(A_{m-1}, A_{m-1}\right)$ and $\left(B C_{m}, B C_{m}\right)$ (see also [10, 11]). In this section, we clarify how our difference operators and kernel functions in the elliptic case are related to those of Ruijsenaars.

In what follows, we confine ourselves to the elliptic case, and set

$$
p=e\left(\omega_{2} / \omega_{1}\right), \quad q=e\left(\delta / \omega_{1}\right) ; \quad[x]=-z^{-\frac{1}{2}} \theta(z ; p), \quad z=e\left(x / \omega_{1}\right)
$$

as in Section 2.1, (2) Elliptic case, assuming that $\operatorname{Im}\left(\omega_{2} / \omega_{1}\right)>0, \operatorname{Im}\left(\delta / \omega_{1}\right)>0$. We remark that the quasi-periodicity of the function $[x]$ is described as

$$
\left[x+\omega_{r}\right]=\epsilon_{r} e\left(\eta_{r}\left(x+\frac{\omega_{r}}{2}\right)\right)[x] \quad(r=0,1,2,3),
$$

where $\omega_{0}=0, \omega_{3}=-\omega_{1}-\omega_{2}$, and

$$
\epsilon_{0}=1, \quad \epsilon_{1}=\epsilon_{2}=\epsilon_{3}=-1 ; \quad \eta_{0}=\eta_{1}=0, \quad \eta_{2}=-\frac{1}{\omega_{1}}, \quad \eta_{3}=\frac{1}{\omega_{1}} .
$$

(We use below the index 0 instead of 4 for $\omega_{r}, \epsilon_{r}$ and $\eta_{r}$.) Since $\prod_{s=1}^{3}\left[\frac{1}{2} \omega_{s}\right]=-2 e\left(-\frac{\omega_{2}}{2 \omega_{1}}\right)=-2 p^{-\frac{1}{2}}$ in this case, we have the duplication formula

$$
[2 x]=2 e\left(\frac{\omega_{2}}{2 \omega_{1}}\right) \prod_{r=0}^{3}\left[x-\frac{\omega_{r}}{2}\right] .
$$

In defining kernel functions, we use the gamma function

$$
G(x \mid \delta)=G_{+}(x \mid \delta)=e\left(\frac{\delta}{2 \omega_{1}}\left(\begin{array}{c}
x / \delta \\
2
\end{array}\right)\right) \Gamma(p z ; p, q)
$$

of $(2.4)$, associated with $[x]$.

In the works of Ruijsenaars [23], the two periods $\omega_{1}, \omega_{2}$ and the scaling constant $\delta$ are parametrized as

$$
\omega_{1}=" \frac{\pi}{r} ", \quad \omega_{2}=" i a_{+} ", \quad \delta=" i a_{-} "
$$

in view of the symmetry between $\omega_{2}$ and $\delta$ (or $p$ and $q$ ). In terms of the $R$-function

$$
\mathcal{R}(x)=" R\left(r, a_{+} ; x\right) "=\theta\left(p^{\frac{1}{2}} z ; p\right)
$$

defined in $[23, \mathrm{I},(1.21)]$, our $[x]$ is expressed as

$$
[x]=-e\left(-\frac{x}{2 \omega_{1}}\right) \mathcal{R}\left(x-\frac{\omega_{2}}{2}\right) .
$$

Note that $\mathcal{R}(-x)=\mathcal{R}(x)$. The elliptic gamma function

$$
\mathcal{G}(x)=" G\left(r, a_{+}, a_{-} ; x\right) "=\Gamma\left(p^{\frac{1}{2}} q^{\frac{1}{2}} z ; p, q\right)
$$

of Ruijsenaars $[23, \mathrm{I},(1.19)]$ is related to our $G(x \mid \delta)$ by the formula

$$
G(x \mid \delta)=e\left(\frac{\delta}{2 \omega_{1}}\left(\begin{array}{c}
x / \delta \\
2
\end{array}\right)\right) \mathcal{G}\left(x+\frac{1}{2}\left(\omega_{2}-\delta\right)\right) .
$$

The function $\mathcal{G}(x)$ is symmetric with respect to $\omega_{2}$ and $\delta(p$ and $q)$, and satisfies the functional equation

$$
\mathcal{G}\left(x+\frac{1}{2} \delta\right)=\mathcal{R}(x) \mathcal{G}\left(x-\frac{1}{2} \delta\right), \quad \mathcal{G}(-x)=\mathcal{G}(x)^{-1} .
$$




\section{B.1 Case of type $A$}

As in (A.1), we consider the commuting family of difference operators $D_{r, x}^{(\delta, \kappa)}(r=1, \ldots, m)$ of type $A$ in the variables $x=\left(x_{1}, \ldots, x_{m}\right)$. These operators are in fact identical to the difference operator $A_{r,+}(-x)$ of $[23, \mathrm{I},(2.1)]$, up to multiplication by constants:

$$
e\left(\frac{r(m-r) \kappa}{2 \omega_{1}}\right) D_{r, x}^{(\delta, \kappa)}=\sum_{|I|=r} \prod_{i \in I ; j \notin I} \frac{\mathcal{R}\left(x_{i}-x_{j}+\kappa-\frac{\omega_{2}}{2}\right)}{\left.\mathcal{R}\left(x_{i}-x_{j}-\frac{\omega_{2}}{2}\right)\right)} \exp \left(\delta \sum_{i \in I} \partial_{x_{i}}\right)=" A_{r,+}(-x) ",
$$

under the identification of the parameter " $\mu$ " $=\kappa$; we denote below this operator by $\mathcal{A}_{r,-x}=$ " $A_{r,+}(-x)$ ". In the multiplicative variables $z_{i}=e\left(x_{i} / \omega_{1}\right)(i=1, \ldots, m), t=e\left(\kappa / \omega_{1}\right)$, the same operator is expressed as

$$
\mathcal{A}_{r,-x}=t^{\frac{1}{2} r(m-r)} D_{r, x}^{(\delta, \kappa)}=\sum_{|I|=r} \prod_{i \in I ; j \notin I} \frac{\theta\left(t z_{i} / z_{j} ; p\right)}{\theta\left(z_{i} / z_{j} ; p\right)} \prod_{i \in I} T_{q, z_{i}} .
$$

The kernel function $\Phi_{A}(x ; y \mid \delta, \kappa)$ of $(2.5)$ for the $m=n$ case can be expressed in terms of $\mathcal{G}(x)$ as

$$
\begin{aligned}
\Phi_{A}(x ; y \mid \delta, \kappa) & =e\left(-\frac{m \kappa}{2 \omega_{1} \delta}\left(\sum_{j=1}^{m} x_{j}+\sum_{l=1}^{m} y_{l}\right)+\frac{m^{2}}{4 \omega_{1} \delta} \kappa(\kappa+\delta-2 v)\right) \mathcal{S}_{A}(-x ; y), \\
\mathcal{S}_{A}(-x ; y) & =\prod_{j, l=1}^{m} \frac{\mathcal{G}\left(-x_{j}-y_{l}-v+\frac{1}{2}\left(\delta-\omega_{2}\right)\right)}{\mathcal{G}\left(-x_{j}-y_{l}-v+\kappa+\frac{1}{2}\left(\delta-\omega_{2}\right)\right)} \\
& =\prod_{j, l=1}^{m} \frac{\Gamma\left(p c z_{j} w_{l} / t ; p, q\right)}{\Gamma\left(p c z_{j} w_{l} ; p, q\right)} \quad\left(c=e\left(v / \omega_{1}\right)\right) .
\end{aligned}
$$

The second factor $\mathcal{S}_{A}(-x ; y)$ of $\Phi_{A}(x ; y \mid \delta, \kappa)$ coincides with " $\mathcal{S}_{\xi}(-x, y)$ " of $[23, \mathrm{I},(2.6)]$, under the identification of parameters " $\mu$ " $=\kappa$ and " $\xi "=-v+\frac{1}{2}\left(\delta+\kappa-\omega_{2}\right)$. Since the first factor of $\Phi_{A}(x ; y \mid \delta, \kappa)$ is an eigenfunction of $T_{x_{i}}^{\delta}$ and $T_{y_{k}}^{\delta}$ with an equal eigenvalue $e\left(-m \kappa / 2 \omega_{1}\right)$, from (A.2) we obtain $\mathcal{A}_{r,-x} \mathcal{S}_{A}(-x ; y)=\mathcal{A}_{r,-y} \mathcal{S}_{A}(-x ; y)$, namely

$$
\mathcal{A}_{r, x} \mathcal{S}_{A}(x ; y)=\mathcal{A}_{r,-y} \mathcal{S}_{A}(x ; y) \quad(r=1, \ldots, m) .
$$

This gives formula $(2.5)$ in $[23, \mathrm{I}]$ with " $\delta=+$ ". The statement for " $\delta=-$ " follows from the symmetry of $\mathcal{S}_{A}(x ; y)$ between $\omega_{2}$ and $\delta$ (or $p$ and $q$ ).

\section{B.2 Case of type $B C$}

We now consider the difference operator $E_{x}^{(\mu \mid \delta, \kappa)}$ of type $B C_{m}$, defined by $(2.7),(2.10),(2.11)$ with the function $[x]$ of (B.1). For the variables $x=\left(x_{1}, \ldots, x_{m}\right)$ and the parameters $\mu=$ $\left(\mu_{1}, \ldots, \mu_{8}\right), \kappa$, we will use the multiplicative expressions together by setting

$$
z_{i}=e\left(x_{i} / \omega_{1}\right) \quad(i=1, \ldots, m), \quad u_{k}=e\left(\mu_{k} / \omega_{1}\right) \quad(k=1, \ldots, 8), \quad t=e\left(\kappa / \omega_{1}\right) .
$$

We first remark that, by $(2.13)$, the difference operator $E_{x}^{(\mu \mid \delta, \kappa)}$ can be rewritten in the form

$$
\begin{aligned}
& t^{m-1} p^{-1} q^{-\frac{3}{2}}\left(u_{1} \cdots u_{8}\right)^{\frac{1}{2}} E_{x}^{(\mu \mid \delta, \kappa)} \\
& \quad=\sum_{1 \leq i \leq m ; \epsilon= \pm}\left(q z_{i}^{\epsilon 2}\right)^{-1} \mathcal{A}_{i}^{\epsilon}(x ; \mu \mid \delta, \kappa) T_{x_{i}}^{\epsilon \delta}+\sum_{r=0}^{3} \mathcal{A}_{r}^{0}(x ; \mu \mid \delta, \kappa) .
\end{aligned}
$$


Here the coefficients $\mathcal{A}_{i}^{ \pm}(x ; \mu \mid \delta, \kappa)(i=1, \ldots, m), \mathcal{A}_{r}^{0}(x ; \mu \mid \delta, \kappa)(r=0,1,2,3)$ are obtained as follows by modifying the corresponding coefficients of $E_{x}^{(\mu \mid \delta, \kappa)}$ :

$$
\begin{aligned}
\mathcal{A}_{i}^{+}(x ; \mu \mid \delta, \kappa)= & \frac{\prod_{k=1}^{8} \mathcal{R}\left(x_{i}+\mu_{k}-\frac{\omega_{2}}{2}\right)}{\mathcal{R}\left(2 x_{i}-\frac{\omega_{2}}{2}\right) \mathcal{R}\left(2 x_{i}+\delta-\frac{\omega_{2}}{2}\right)} \prod_{1 \leq j \leq m ; j \neq i} \frac{\mathcal{R}\left(x_{i} \pm x_{j}+\kappa-\frac{\omega_{2}}{2}\right)}{\mathcal{R}\left(x_{i} \pm x_{j}-\frac{\omega_{2}}{2}\right)} \\
= & \frac{\prod_{k=1}^{8} \theta\left(u_{k} z_{i} ; p\right)}{\theta\left(z_{i}^{2} ; p\right) \theta\left(q z_{i}^{2} ; p\right)} \prod_{1 \leq j \leq m ; j \neq i} \frac{\theta\left(t z_{i} z_{j}^{ \pm 1} ; p\right)}{\theta\left(z_{i} z_{j}^{ \pm 1} ; p\right)}, \\
\mathcal{A}_{i}^{-}(x ; \mu \mid \delta, \kappa)= & \mathcal{A}_{i}^{+}(-x ; \mu \mid \delta, \kappa) \quad(i=1, \ldots, m), \\
\mathcal{A}_{r}^{0}(x ; \mu \mid \delta, \kappa)= & C_{r} \frac{\prod_{k=1}^{8} \mathcal{R}\left(\mu_{k}+\frac{\omega_{r}}{2}-\frac{1}{2}\left(\omega_{2}+\delta\right)\right)}{2 \mathcal{R}\left(\kappa-\frac{\omega_{2}}{2}\right) \mathcal{R}\left(\kappa-\delta-\frac{\omega_{2}}{2}\right)} \prod_{j=1}^{m} \frac{\mathcal{R}\left( \pm x_{j}+\kappa+\frac{\omega_{r}}{2}-\frac{1}{2}\left(\omega_{2}+\delta\right)\right)}{\mathcal{R}\left( \pm x_{j}+\frac{\omega_{r}}{2}-\frac{1}{2}\left(\omega_{2}+\delta\right)\right)} \\
= & C_{r} \frac{\prod_{k=1}^{8} \theta\left(u_{k} q^{-\frac{1}{2}} c_{r} ; p\right)}{2 \theta(t ; p) \theta\left(t q^{-1} ; p\right)} \prod_{j=1}^{m} \frac{\theta\left(t q^{-\frac{1}{2}} c_{r} z_{j}^{ \pm 1} ; p\right)}{\theta\left(q^{-\frac{1}{2}} c_{r} z_{j}^{ \pm 1} ; p\right)} \quad(r=0,1,2,3)
\end{aligned}
$$

with constants

$$
c_{r}=e\left(\omega_{r} / 2 \omega_{1}\right), \quad C_{r}=e\left(-\frac{2 \omega_{r}}{\omega_{1}}-\eta_{r}\left(\omega_{r}-2 \delta+m \kappa+\frac{1}{2} \sum_{k=1}^{8} \mu_{k}\right)\right) \quad(r=0,1,2,3) .
$$

In the multiplicative expression, these constants are expressed as

$$
\begin{aligned}
& c_{0}=1, \quad c_{1}=-1, \quad c_{2}=p^{\frac{1}{2}}, \quad c_{3}=-p^{-\frac{1}{2}} ; \\
& C_{0}=C_{1}=1, \quad C_{2}=t^{m} p^{-1} q^{-2}\left(u_{1} \cdots u_{8}\right)^{\frac{1}{2}}, \quad C_{3}=t^{-m} p^{3} q^{2}\left(u_{1} \cdots u_{8}\right)^{-\frac{1}{2}} .
\end{aligned}
$$

In view of formula (B.2), we take a nonzero meromorphic function $P(x)$ satisfying the system of difference equations

$$
T_{x_{i}}^{\delta}(P(x))=q z_{i}^{2} P(x) \quad(i=1, \ldots, m) .
$$

The simplest choice for such a function is given by $P(x)=e\left(\frac{1}{\omega_{1} \delta} \sum_{j=1}^{m} x_{j}^{2}\right)$. Then formula (B.2) implies that the difference operator $E_{x}^{(\mu \mid \delta, \kappa)}$ is expressed in the form

$$
E_{x}^{(\mu \mid \delta, \kappa)}=t^{-m+1} p q^{\frac{3}{2}}\left(u_{1} \cdots u_{8}\right)^{-\frac{1}{2}} P(x) \mathcal{E}_{x}^{(\mu \mid \delta, \kappa)} P(x)^{-1},
$$

as a constant multiple of the conjugation of a difference operator

$$
\mathcal{E}_{x}^{(\mu \mid \delta, \kappa)}=\sum_{1 \leq i \leq m ; \epsilon= \pm} \mathcal{A}_{i}^{\epsilon}(x ; \mu \mid \delta, \kappa) T_{x_{i}}^{\epsilon \delta}+\sum_{r=0}^{3} \mathcal{A}_{r}^{0}(x ; \mu \mid \delta, \kappa)
$$

by $P(x)$.

We also remark that the operator $\mathcal{E}_{x}^{(\mu \mid \delta, \kappa)}$ has the following property with respect to shifting $\kappa$ to $\kappa-\omega_{2}(t$ to $t / p)$ :

$$
\mathcal{E}_{x}^{(\mu \mid \delta, \kappa)}=\left(p^{2} q t^{-2}\right)^{m-1} P(x)^{m-1} \mathcal{E}_{x}^{\left(\mu \mid \delta, \kappa-\omega_{2}\right)} P(x)^{-m+1}, \quad t=e\left(\kappa / \omega_{1}\right) .
$$


Together with (B.3), this also implies

$$
E_{x}^{(\mu \mid \delta, \kappa)}=t^{-3(m-1)} p^{2 m-1} q^{m+\frac{1}{2}}\left(u_{1} \cdots u_{8}\right)^{-\frac{1}{2}} P(x)^{m} \mathcal{E}_{x}^{\left(\mu \mid \delta, \kappa-\omega_{2}\right)} P(x)^{-m} .
$$

Setting $\kappa=\lambda+\omega_{2}\left(t=s p, s=e\left(\lambda / \omega_{1}\right)\right)$, we rewrite this formula as

$$
E_{x}^{\left(\mu \mid \delta, \lambda+\omega_{2}\right)}=s^{-3(m-1)} p^{-m+2} q^{m+\frac{1}{2}}\left(u_{1} \cdots u_{8}\right)^{-\frac{1}{2}} P(x)^{m} \mathcal{E}_{x}^{(\mu \mid \delta, \lambda)} P(x)^{-m} .
$$

The difference operator $\mathcal{E}_{x}^{(\mu \mid \delta, \lambda)}$ appearing in (B.4) is essentially the same as the operator " $A_{+}(h, \mu ; x)$ " of type $B C_{m}$ defined by Ruijsenaars [23, I, (4.1)-(4.3)] (see also (3.1)-(3.9)); the difference between the two is only by an additive constant. In fact, we have

$$
" A_{+}(h, \mu ; x) "=\mathcal{E}_{x}^{(\mu \mid \delta, \lambda)}-\sum_{r=0}^{3} \mathcal{A}_{r}^{0}\left(\xi_{r}, \ldots, \xi_{r} ; \mu \mid \delta, \lambda\right),
$$

where $\xi_{0}=\xi_{2}=\omega_{1} / 2, \xi_{1}=\xi_{3}=0$, under the identification of the parameters

$$
" h_{k} "=\mu_{k}-\frac{1}{2}\left(\omega_{2}+\delta\right) \quad(k=0,1, \ldots, 7), \quad " \mu "=\lambda,
$$

with $\mu_{0}=\mu_{8}$.

By Theorem 2.3 we know that the kernel function of (2.15)

$$
\Phi_{B C}(x ; y \mid \delta, \kappa)=\prod_{j=1}^{m} \prod_{l=1}^{n} \prod_{\epsilon_{1}, \epsilon_{2}= \pm} G\left(\epsilon_{1} x_{j}+\epsilon_{2} y_{l}+\frac{1}{2}(\delta-\kappa) \mid \delta\right)
$$

in the variables $x=\left(x_{1}, \ldots, x_{m}\right)$ and $y=\left(y_{1}, \ldots, y_{n}\right)$ satisfies the difference equation

$$
\begin{aligned}
& E_{x}^{(\mu \mid \delta, \kappa)} \Phi_{B C}(x ; y \mid \delta, \kappa)=E_{y}^{(\widetilde{\mu} \mid \delta, \kappa)} \Phi_{B C}(x ; y \mid \delta, \kappa), \\
& \tilde{\mu}_{k}=\frac{1}{2}(\delta+\kappa)-\mu_{k} \quad(k=1, \ldots, 8)
\end{aligned}
$$

under the balancing condition

$$
(m-n-1) \kappa-\delta+\frac{1}{2} \sum_{k=1}^{8} \mu_{k}=0 \quad\left(t^{m-n-1} q^{-1}\left(u_{1} \cdots u_{8}\right)^{\frac{1}{2}}=1\right) .
$$

With $G(x \mid \delta)=G_{+}(x \mid \delta),(2.4)$, this kernel function is given explicitly by

$$
\Phi_{B C}(x ; y \mid \delta, \kappa)=e\left(\frac{f(x ; y)}{\omega_{1} \delta}\right) \prod_{j=1}^{m} \prod_{l=1}^{n} \prod_{\epsilon_{1}, \epsilon_{2}= \pm} \Gamma\left(p q^{\frac{1}{2}} t^{-\frac{1}{2}} z_{j}^{\epsilon_{1}} w_{l}^{\epsilon_{2}} ; p, q\right)
$$

where $w_{l}=e\left(y_{l} / \omega_{1}\right)(l=1, \ldots, n)$, and $f(x ; y)=n \sum_{j=1}^{m} x_{j}^{2}+m \sum_{l=1}^{n} y_{l}^{2}+\frac{m n}{4}\left(\kappa^{2}-\delta^{2}\right)$. We express $\Phi_{B C}(x ; y \mid \delta, \kappa)$ in the form

$$
\Phi_{B C}(x ; y \mid \delta, \kappa)=\mathrm{const} \cdot P(x)^{n} P(y)^{m} \mathcal{S}_{B C}(x ; y),
$$

where

$$
P(x)=e\left(\frac{1}{\omega_{1} \delta} \sum_{j=1}^{m} x_{j}^{2}\right), \quad P(y)=e\left(\frac{1}{\omega_{1} \delta} \sum_{l=1}^{n} y_{l}^{2}\right),
$$




$$
\mathcal{S}_{B C}(x ; y)=\prod_{j=1}^{m} \prod_{l=1}^{n} \prod_{\epsilon_{1}, \epsilon_{2}= \pm} \Gamma\left(p q^{\frac{1}{2}} t^{-\frac{1}{2}} z_{j}^{\epsilon_{1}} w_{l}^{\epsilon_{2}} ; p, q\right) .
$$

It should be noted that, by the substitution $\kappa=\lambda+\omega_{2}\left(t=s p, s=e\left(\lambda / \omega_{1}\right)\right)$, the function

$$
\mathcal{S}_{B C}(x ; y)=\prod_{j=1}^{m} \prod_{l=1}^{n} \prod_{\epsilon_{1}, \epsilon_{2}= \pm} \Gamma\left(p^{\frac{1}{2}} q^{\frac{1}{2}} s^{-\frac{1}{2}} z_{j}^{\epsilon_{1}} w_{l}^{\epsilon_{2}} ; p, q\right)=\prod_{j=1}^{m} \prod_{l=1}^{n} \prod_{\epsilon_{1}, \epsilon_{2}= \pm} \mathcal{G}\left(\epsilon_{1} x_{j}+\epsilon_{2} y_{l}-\frac{1}{2} \lambda\right)
$$

becomes manifestly invariant under the action of the Weyl group of type $B C_{m}$ (resp. $B C_{n}$ ) on the variables $x=\left(x_{1}, \ldots, x_{m}\right)$ (resp. $\left.y=\left(y_{1}, \ldots, y_{n}\right)\right)$, and symmetric with respect to $\omega_{2}$ and $\delta$ $(p$ and $q)$. This function $\mathcal{S}_{B C}(x ; y)$ can be thought of as the $\left(B C_{m}, B C_{n}\right)$-version of Ruijsenaars' kernel function " $\mathcal{S}(x ; y)$ " in [23].

We now have the functional equation

$$
E_{x}^{(\mu \mid \delta, \kappa)} P(x)^{n} P(y)^{m} \mathcal{S}_{B C}(x ; y)=E_{y}^{(\widetilde{\mu} \mid \delta, \kappa)} P(x)^{n} P(y)^{m} \mathcal{S}_{B C}(x ; y)
$$

under the balancing condition (B.6). Hence, by applying (B.4) with $\kappa=\lambda+\omega_{2}$, we obtain

$$
\begin{aligned}
\text { const } & \cdot P(x)^{m} \mathcal{E}_{x}^{(\mu \mid \delta, \lambda)} P(x)^{n-m} P(y)^{m} \mathcal{S}_{B C}(x ; y) \\
= & \text { const } \cdot P(y)^{n} \mathcal{E}_{y}^{(\widetilde{\mu} \mid \delta, \lambda)} P(x)^{n} P(y)^{m-n} \mathcal{S}_{B C}(x ; y),
\end{aligned}
$$

namely,

$$
\begin{aligned}
\text { const } & \cdot \mathcal{E}_{x}^{(\mu \mid \delta, \lambda)} P(x)^{n-m} P(y)^{m-n} \mathcal{S}_{B C}(x ; y) \\
= & \text { const } \cdot \mathcal{E}_{y}^{(\widetilde{\mu} \mid \delta, \lambda)} P(x)^{n-m} P(y)^{m-n} \mathcal{S}_{B C}(x ; y) .
\end{aligned}
$$

The constants in front of the both sides simplifies by (B.6), to imply the functional equation

$$
\begin{aligned}
& (p q / s)^{m} \mathcal{E}_{x}^{(\mu \mid \delta, \lambda)} P(x)^{n-m} P(y)^{m-n} \mathcal{S}_{B C}(x ; y) \\
& \quad=(p q / s)^{n} \mathcal{E}_{y}^{(\widetilde{\mu} \mid \delta, \lambda)} P(x)^{n-m} P(y)^{m-n} \mathcal{S}_{B C}(x ; y),
\end{aligned}
$$

under the balancing condition

$$
\frac{1}{2} \sum_{k=1}^{8} \mu_{k}=\delta-(m-n-1)\left(\lambda+\omega_{2}\right) \quad\left(\left(u_{1} \cdots u_{8}\right)^{\frac{1}{2}}=q(p s)^{-m+n+1}\right) .
$$

In particular, when $m=n$ we have

$$
\mathcal{E}_{x}^{(\mu \mid \delta, \lambda)} \mathcal{S}_{B C}(x ; y)=\mathcal{E}_{y}^{(\widetilde{\mu} \mid \delta, \lambda)} \mathcal{S}_{B C}(x ; y),
$$

under the balancing condition

$$
\frac{1}{2} \sum_{k=1}^{8} \mu_{k}=\lambda+\omega_{2}+\delta \quad\left(\left(u_{1} \cdots u_{8}\right)^{\frac{1}{2}}=s p q\right) .
$$

The functional equation (B.7) is precisely the formula (4.24) of [23, I, Proposition 4.1], for " $\delta=+$ " with the parameter

$$
" h_{k} "=\mu_{k}-\frac{1}{2}\left(\omega_{2}+\delta\right) \quad(k=0,1, \ldots, 7), \quad \text { " } "=\lambda .
$$

The balancing condition (B.8) corresponds to condition (4.26), “ $\mu=2 i a+\frac{1}{2} \sum_{k=0}^{7} h_{k}$ ". Also the parameters $\widetilde{\mu}_{k}=\frac{1}{2}\left(\lambda+\omega_{2}+\delta\right)-\mu_{k}(k=1, \ldots, 8)$ for the $y$ variables are consistent with " $\left(-J_{R} h\right)_{k}=\frac{\mu}{2}-i a-h_{k}$ " $(k=0,1, \ldots, 7)$. (The constant " $\sigma_{+}(h)$ " on the right side of [23, I, (4.24)], arises as the difference of constant terms of two operators, as indicated in (B.5).) 


\section{C $\quad E_{r}(z ; a \mid t)$ and $H_{l}(z ; a \mid q, t)$ as interpolation polynomials}

In Section 5 we presented some explicit expansion formulas for Koornwinder polynomials attached to single columns and to single rows, in terms of invariant Laurent polynomials $E_{r}(z ; a \mid t)$ and $H_{l}(z ; a \mid q, t)$, respectively. These Laurent polynomials $E_{r}(z ; a \mid t)$ and $H_{l}(z ; a \mid q, t)$ are essentially the same objects as the $B C_{m}$-interpolation polynomials of Okounkov [18] attached to single columns and single rows. Our Theorems 5.1 and 5.2 provides with explicit expressions for the corresponding special cases of the binomial expansion of Koornwinder polynomials in terms of $B C_{m}$-interpolation polynomials as discussed in Okounkov [18] and Rains [19].

In the notation of Section 5 , the $B C_{m}$ interpolation polynomial $P_{\lambda}^{*}(z ; q, t, a)$ attached a partition $\lambda=\left(\lambda_{1}, \ldots, \lambda_{m}\right)$ [18] is characterized uniquely, up to multiplication by a constant, as a Laurent polynomial in $\mathbb{K}\left[z^{ \pm}\right]$of degree $|\lambda|$ satisfying the following conditions:

(0) $P_{\lambda}^{*}(z ; q, t, a)$ is $W$-invariant in the shifted variables $z t^{\delta} a=\left(z_{1} t^{m-1} a, z_{2} t^{m-2} a, \ldots, z_{m} a\right)$, where $\delta=(m-1, m-2 \ldots, 0)$.

(1) $P_{\lambda}^{*}\left(q^{\mu} ; q, t, a\right)=0$ for any partition $\mu=\left(\mu_{1}, \ldots, \mu_{m}\right)$ such that $\mu \not \supset \lambda$.

(2) $P_{\lambda}^{*}\left(q^{\lambda} ; q, t, a\right) \neq 0$.

In this section we show that the Laurent polynomials $E_{r}\left(z t^{\delta} a ; a \mid t\right)$ and $H_{l}\left(z t^{\delta} a ; a \mid q, t\right)$ coincide, up to constant multiplication, with the interpolation polynomials $P_{\left(1^{r}\right)}^{*}(z ; q, t, a)$ and $P_{(l)}^{*}(z ; q, t, a)$, respectively. We prove that these polynomials actually have the interpolation properties as mentioned above.

Proposition C.1. For each $r=0,1, \ldots, m$, let $E_{r}(z ; a \mid t)$ be the $W$-invariant Laurent polynomial defined in (5.1).

(0) $E_{r}\left(z_{1}, \ldots, z_{r}, t^{m-r-1} a, \ldots, a ; a \mid t\right)=\left\langle z_{1} ; t^{m-r} a\right\rangle \cdots\left\langle z_{r} ; t^{m-r} a\right\rangle$.

(1) For any partition $\mu$ with $l(\mu)<r$, i.e., $\mu \not \supset\left(1^{r}\right), E_{r}\left(q^{\mu} t^{\delta} a ; a \mid t\right)=0$.

(2) $E_{r}\left(q t^{m-1} a, \ldots, q t^{m-r} a, t^{m-r-1} a, \ldots, a ; a \mid t\right)=(-1)^{r}\left\langle t^{m-r} a ; q t^{m-r} a\right\rangle_{t, r}$.

Proof. Since $E_{r}(z ; a \mid t)$ is symmetric in $z=\left(z_{1}, \ldots, z_{m}\right)$, from the recurrence relation (5.5) we have

$$
E_{r}(z ; a \mid t)=E_{r}\left(z_{1}, \ldots, z_{m-1} ; t a \mid t\right)+E_{r-1}\left(z_{1}, \ldots, z_{m-1} ; a \mid t\right)\left\langle z_{m} ; a\right\rangle
$$

and hence by setting $z_{m}=a$,

$$
E_{r}\left(z_{1}, \ldots, z_{m-1}, a ; a \mid t\right)=E_{r}\left(z_{1}, \ldots, z_{m-1} ; t a \mid t\right) .
$$

This implies

$$
\begin{aligned}
E_{r}\left(z_{1}, \ldots, z_{r}, t^{m-r-1} a, \ldots, a ; a \mid t\right) & =E_{r}\left(z_{1}, \ldots, z_{r}, t^{m-r-1} a, \ldots, t a ; t a \mid t\right)=\cdots \\
& =E_{r}\left(z_{1}, \ldots, z_{r} ; t^{m-r} a \mid t\right)=\left\langle z_{1} ; t^{m-r} a\right\rangle \cdots\left\langle z_{r} ; t^{m-r} a\right\rangle
\end{aligned}
$$

for $r=0,1, \ldots, m$, which proves $(0)$. In particular, we have

$$
E_{r}\left(z_{1}, \ldots, z_{r-1}, t^{m-r} a, t^{m-r-1} a, \ldots, a ; a \mid t\right)=0,
$$

which implies $E_{r}\left(q^{\mu} t^{\delta} a ; a \mid t\right)=0$ for any partition such that $l(\mu)<r$, namely, $\mu \not \supset\left(1^{r}\right)$. Statement (0) also implies

$$
E_{r}\left(q t^{m-1} a, \ldots, q t^{m-r} a, t^{m-r-1} a, \ldots, a ; a \mid t\right)=(-1)^{r}\left\langle t^{m-r} a ; q t^{m-r} a\right\rangle_{t, r} .
$$


From this proposition we see that $E_{r}\left(z t^{\delta} a ; a \mid t\right)$ is a constant multiple of the interpolation polynomial $P_{\left(1^{r}\right)}^{*}(z ; q, t, a)$ for each $r=0,1, \ldots, m$.

Proposition C.2. For $l=0,1,2, \ldots$, let $H_{l}(z ; a \mid q, t)$ be the $W$-invariant Laurent polynomial defined in (5.3).

(1) For any partition $\mu=\left(\mu_{1}, \ldots, \mu_{m}\right)$ with $\mu_{1}<l$, i.e., $\mu \not \supset(l), H_{l}\left(q^{\mu} t^{\delta} a ; a \mid q, t\right)=0$.

(2) $H_{l}\left(q^{l} t^{m-1} a, t^{m-2} a, \ldots, a ; a \mid q, t\right)=\langle t\rangle_{q, l}\left\langle q^{l} t^{2(m-1)} a^{2}\right\rangle_{q, l}$.

Proof. By the definition (5.3), the Laurent polynomials $H_{l}(z ; a \mid q, t)(l=0,1,2 \ldots)$ satisfy the recurrence formula

$$
H_{l}(z ; a \mid q, t)=\sum_{r=0}^{l} \frac{\langle t\rangle_{q, l-r}}{\langle q\rangle_{q, l-r}} H_{r}\left(z_{1}, \ldots, z_{m-1} ; a \mid q, t\right)\left\langle z_{m} ; q^{r} t^{m-1} a\right\rangle_{q, l-r} .
$$

Since these Laurent polynomials are symmetric in $z=\left(z_{1}, \ldots, z_{m}\right)$ (Lemma 5.4), we also have

$$
H_{l}(z ; a \mid q, t)=\sum_{r=0}^{l} \frac{\langle t\rangle_{q, l-r}}{\langle q\rangle_{q, l-r}}\left\langle z_{1} ; t^{m-1} q^{r} a\right\rangle_{q, l-r} H_{r}\left(z_{2}, \ldots, z_{m} ; a \mid q, t\right) .
$$

We prove proposition by the induction on $m$. For an arbitrary partition $\mu=\left(\mu_{1}, \ldots, \mu_{m}\right)$, we specialize this recurrence formula to $z=q^{\mu} t^{\delta} a$ :

$$
H_{l}\left(q^{\mu} t^{\delta} a ; a \mid q, t\right)=\sum_{r=0}^{l} \frac{\langle t\rangle_{q, l-r}}{\langle q\rangle_{q, l-r}}\left\langle q^{\mu_{1}} t^{m-1} a ; q^{r} t^{m-1} a\right\rangle_{q, l-r} H_{r}\left(q^{\mu_{2}} t^{m-2} a, \ldots, q^{\mu_{m}} a ; a \mid q, t\right) .
$$

By the induction hypothesis we have

$$
H_{r}\left(q^{\mu_{2}} t^{m-2} a, \ldots, q^{\mu_{m}} a ; a \mid q, t\right)=0
$$

for $r>\mu_{1}$. For $r \leq \mu_{1}$, we see

$$
\left\langle q^{\mu_{1}} t^{m-1} a ; q^{r} t^{m-1} a\right\rangle_{q, l-r}=(-1)^{l-r}\left\langle q^{-\mu_{1}+r}\right\rangle_{q, l-r}\left\langle q^{\mu_{1}+r} t^{2(m-1)} a^{2}\right\rangle_{q, l-r} .
$$

Since $-\mu_{1}+r \leq 0,\left\langle q^{-\mu_{1}+r}\right\rangle_{q, l-r}=0$ if $l-r>\mu_{1}-r$, i.e., $\mu_{1}<l$. This means that all the terms on the right side of (C.1) vanish when $\mu_{1}<l$, and hence, $H_{l}\left(q^{\mu} t^{\delta} a ; a \mid q, t\right)=0$. When $\mu=(l)$, the only nontrivial term arises from $r=0$ :

$$
H_{l}\left(q^{l} t^{m-1} a, t^{m-2} a, \ldots, a ; a \mid q, t\right)=\frac{\langle t\rangle_{q, l}}{\langle q\rangle_{q, l}}\left\langle q^{l} t^{m-1} a ; t^{m-1} a\right\rangle_{q, l}=\langle t\rangle_{q, l}\left\langle q^{l} t^{2(m-1)} a^{2}\right\rangle_{q, l} .
$$

This proposition implies that $H_{l}\left(z t^{\delta} a ; a \mid a, t\right)$ is a constant multiple of the interpolation polynomial $P_{(l)}^{*}(z ; q, t, a)$ for each $l=0,1,2, \ldots$

\section{Acknowledgements}

The authors express their sincere gratitude to Professor Simon Ruijsenaars for his valuable comments concerning the comparison of results in the present paper with those in his series of works, and to Professor Eric Rains for suggesting the connection of the explicit formulas for the Koornwinder polynomials in this paper with the binomial expansion formula in terms of interpolation polynomials. Also, they are grateful to the anonymous referees who kindly offered helpful suggestions for improving the manuscript. 


\section{References}

[1] Aomoto K., Ito M., A determinant formula for a holonomic $q$-difference system associated with Jackson integrals of type $B C_{n}, A d v$. Math., to appear.

[2] van Diejen J.F., Integrability of difference Calogero-Moser systems, J. Math. Phys. 35 (1994), $2983-3004$.

[3] van Diejen J.F., Self-dual Koornwinder-Macdonald polynomials, Invent. Math. 126 (1996), 319-339, q-alg/9507033.

[4] Gasper G., Rahman M., Basic hypergeometric series, 2nd ed., Encyclopedia of Mathematics and Its Applications, Vol. 96, Cambridge University Press, Cambridge, 2004.

[5] Kajihara Y., Noumi M., Raising operators of row type for Macdonald polynomials, Compositio Math. 120 (2000), 119-136, math.QA/9803151.

[6] Kajihara Y., Noumi M., Multiple elliptic hypergeometric series. An approach from the Cauchy determinant, Indag. Math. (N.S.) 14 (2003), 395-421, math.CA/0306219.

[7] Kirillov A.N., Noumi M., Affine Hecke algebras and raising operators for Macdonald polynomials, Duke Math. J. 93 (1998), 1-39, q-alg/9605004.

[8] Kirillov A.N., Noumi M., q-difference raising operators for Macdonald polynomials and the integrality of transition coefficients, in Algebraic Methods and $q$-Special Functions (Montréal, QC, 1996), CRM Proc. Lecture Notes, Vol. 22, Amer. Math. Soc., Providence, RI, 1999, 227-243, q-alg/9605005.

[9] Komori Y., Hikami K., Quantum integrability of the generalized elliptic Ruijsenaars models, J. Phys. A: Math. Gen. 30 (1997), 4341-4364.

[10] Koornwinder T.H., Askey-Wilson polynomials for root systems of type $B C$, in Hypergeometric Functions on Domains of Positivity, Jack Polynomials, and Applications (Tampa, FL, 1991), Contemp. Math., Vol. 138, Amer. Math. Soc., Providence, RI, 1992, 189-204.

[11] Lassalle M., Some conjectures for Macdonald polynomials of type B, C, D, Sém. Lothar. Combin. 52 (2004), Art. B52h, 24 pages, math.CO/0503149.

[12] Langmann E., An explicit solution of the (quantum) elliptic Calogero-Sutherland model, in SPT 2004Symmetry and Perturbation Theory, World Sci. Publ., Hackensack, NJ, 2005, 159-174, math-ph/0407050.

[13] Langmann E., Remarkable identities related to the (quantum) elliptic Calogero-Sutherland model, J. Math. Phys. 47 (2006), 022101, 18 pages, math-ph/0406061.

[14] Macdonald I.G., Symmetric functions and Hall polynomials, 2nd ed., Oxford University Press, New York, 1995.

[15] Mimachi K., A duality of Macdonald-Koornwinder polynomials and its applications to integral representations, Duke Math. J. 107 (2001), 263-281.

[16] Mimachi K., Noumi M., An integral representation of eigenfunctions for Macdonald's $q$-difference operators, Tôhoku Math. J. 49 (1997), 517-525.

[17] Mimachi K., Noumi M., A reproducing kernel for nonsymmetric Macdonald polynomials, Duke Math. J. 91 (1998), 621-634, q-alg/9610014.

[18] Okounkov A., BC-type interpolation Macdonald polynomials and binomial formula for Koornwinder polynomials, Transform. Groups 3 (1998), 181-207, q-alg/9611011.

[19] Rains E.M., $B C_{n}$-symmetric polynomials, Transform. Groups 10 (2005), 63-132, math.QA/0112035.

[20] Ruijsenaars S.N.M., Complete integrability of relativistic Calogero-Moser systems and elliptic function identities, Comm. Math. Phys. 110 (1987), 191-213.

[21] Ruijsenaars S.N.M., Elliptic integrable systems of Calogero-Moser type: some new results on joint eigenfunctions, in Elliptic Integrable Systems, Proceedings of the Workshop on Elliptic Integrable Systems (Kyoto, 2004), Rokko Lectures in Mathematics, Vol. 18, Kobe University, 2005, 223-240.

[22] Ruijsenaars S.N.M., Eigenfunctions with a zero eigenvalue for differences of elliptic relativistic CalogeroMoser Hamiltonians, Theoret. and Math. Phys. 146 (2006), 25-33.

[23] Ruijsenaars S.N.M., Hilbert-Schmidt operators vs. integrable systems of elliptic Calogero-Moser type. I. The eigenfunction identities, Comm. Math. Phys. 286 (2009), 629-657.

Ruijsenaars S.N.M., Hilbert-Schmidt operators vs. integrable systems of elliptic Calogero-Moser type. II. The $A_{N-1}$ case: first steps, Comm. Math. Phys. 286 (2009), 659-680.

[24] Stokman J.V., Lecture notes on Koornwinder polynomials, in Laredo Lectures on Orthogonal Polynomials and Special Functions, Adv. Theory Spec. Funct. Orthogonal Polynomials, Nova Sci. Publ., Hauppauge, NY, 2004, 145-207. 